\title{
Stratégie foncière de l'habitant : Québec (1790-1835)
}

\section{Gilles Paquet et Jean-Pierre Wallot}

Volume 39, numéro 4, printemps 1986

URI : https://id.erudit.org/iderudit/304401ar

DOI : https://doi.org/10.7202/304401ar

Aller au sommaire du numéro

Éditeur(s)

Institut d'histoire de l'Amérique française

ISSN

0035-2357 (imprimé)

1492-1383 (numérique)

Découvrir la revue

Citer cet article

Paquet, G. \& Wallot, J.-P. (1986). Stratégie foncière de l'habitant : Québec (1790-1835). Revue d'histoire de l'Amérique française, 39(4), 551-581.

https://doi.org/10.7202/304401ar d'utilisation que vous pouvez consulter en ligne.

https://apropos.erudit.org/fr/usagers/politique-dutilisation/ 


\title{
STRATÉGIE FONCIERE DE L'HABITANT: QUÉBEC $(\mathbf{1 7 9 0 - 1 8 3 5})^{1}$
}

\author{
GILLES PAQUET \\ Université d'Ottawa \\ JEAN-PIERRE WALLOT \\ Archives publiques du Canada
}

«...they love money and are seldom on the wrong side of a bargain.» John Lambert (1810)

\section{Introduction}

Le chromo ${ }^{2}$ qu'on a produit pour représenter l'habitant ${ }^{3}$ du Québec du $17 \mathrm{e}$ au $19 \mathrm{e}$ siècle est bien connu. Il a été repris un peu partout dans l'historiographie tant canadienne-française que canadienneanglaise. L'habitant y apparaît comme un paysan ignorant, conservateur, «traditionnel» et peu sensible aux signaux du marché. C'est un être d'Ancien Régime. De ce conservatisme présumé, nombre d'historiens, et à leur suite des sociologues, ont dérivé toute une série de conséquences qui «expliqueraient» la plupart des problèmes socio-économiques du Québec aux 18e et 19e siècles: surpeuplement, morcellement constant du sol et des héritages, surexploitation de terres de plus en plus petites avec des techniques primitives, chute des rendements agricoles, appauvrissement continu de la masse rurale - ces éléments constituant les fondements d'une crise agricole qui aurait frappé le Québec au tout début du $19 \mathrm{e}$ siècle ${ }^{4}$.

1 Texte remanié et augmenté d'une communication à un symposium de la Société Royale du Canada (Guelph, mai 1984). Nous remercions George Bervin et Henriette Nicoll pour leur assistance, le Conseil de recherche en sciences humaines, pour ses subventions, et Serge Courville, pour ses commentaires et conseils toujours utiles.

Le Petit Robert (édition 1977, 312), définit le mot chromo ainsi: «toute image en couleur de mauvais goût».

3 Par habitant, nous entendons l'agriculteur ou le cultivateur canadien, presque toujours propriétaire d'une superficie variable de terre qui se situe le plus souvent entre 60 et 120 arpents, au début du $19 \mathrm{e}$ siècle. Il arrive parfois que l'habitant soit locataire, mais en général il possède sa terre, ses animaux, ses stocks et ses instruments aratoires. Une certaine imprécision émerge dans les années 1820-1850, alors qu'un nombre croissant de fils d'habitants, parfois surnommés euxmêmes habitants, habitent les villages qui se multiplient rapidement à cette époque et vivent d'un métier ou encore de divers travaux comme manoeuvres ( $\mathrm{S}$. Courville, «Esquisse du développement villageois au Québec: le cas de l'aire seigneuriale entre 1760 et 1854», Cahiers de géographie du Québec (CGQ), 28 (1984): 9-46).

4 L'idée d'une crise agricole au Bas-Canada au cours du premier tiers du $19 \mathrm{e}$ siècle, est assez ancienne. Mais c'est F. Ouellet et J. Hamelin ( «La crise agricole dans le Bas-Canada, 180237», Rapport annuel de la Société Historique du Canada (Ottawa, 1962), 17-33) qui placent cette crise au centre de l'explication de l'évolution du Bas-Canada dans la première moitié du 19e siècle. Cette thèse a été reprise et amplifiée par F. Ouellet en 1966 (Histoire économique et sociale 
Cette perspective devait inspirer tout un ensemble de travaux qui ont proposé une explication culturelle à une série de phénomènes allant des Rébellions de 1837 à la productivité différentielle des agricultures québécoise et ontarienne au $19 \mathrm{e}$ siècle, au retard économique des Canadiens français en général et à l'anémie de leur entrepreneurship jusqu'au milieu du 20e siècle 5 .

A notre avis, ce chromo ne correspond guère à la réalité observée et les explications culturelles qui s'y aboutent ne réussissent pas à convaincre. Pourtant, il semble indélébile et l'historiographie, même récente, continue souvent à en colporter l'image.

La section 2 dégage les caractéristiques principales de ce chromo classique et les argumentations qui en ont découlé. Puis elle esquisse une image de rechange de l'habitant québécois qui respecte mieux le sens commun et les faits. Dans la section 3, cette image de rechange nous permet d'ausculter la stratégie économique des habitants. Les sections 4 et 5 présentent les données utiles à une première analyse du pattern d'accumulation du capital foncier dans la région de Québec, entre 1790 et 1835, à la lueur de ce schéma de rechange. La conclusion suggère quelques pistes de recherche.

\section{Le chromo et le modèle}

L'histoire, comme les autres sciences de l'homme, est une science inexacte et le gros de ses résultats reste au niveau des conjectures. Mais le caractère inexact des sciences humaines ne les condamne pas pour autant à l'arbitraire. Il existe des techniques susceptibles de rendre ces travaux moins imparfaits: par exemple, une problématique explicitée (Chaunu) et un recours judicieux à la méthode scientifique (Rescher) ${ }^{6}$. Jusqu'au milieu du 20e siècle, ces impératifs ont été trop souvent ignorés

\footnotetext{
du Québec, 1760-1850 (Montréal, 1966), puis en 1972 (Éléments d'histoire sociale du BasCanada (Montréal, 1972). Même si le simplisme de l'explication semble s'atténuer (Le BasCanada 1791-1840 (Ottawa, 1976), ce n'est que pour reparaître encore plus fortement par après (exemple, «Le mythe de l'habitant sensible au marché», Recherches sociographiques, 17 (1976): 115-132). Pour une critique de la position ouellettiste, voir G. Paquet et J.-P. Wallot, «Crise agricole et tensions socio-ethniques dans le Bas-Canada, 1802-1812», Revue d' histoire de l'Amérique française (RHAF), 26,2 (septembre 1972): 239-270, et «The Agricultural Crisis in Lower Canada, 1802-1812: mise au point», Canadian Historical Review (CHR), 56 (1975): 134-168. Voir aussi note 16 .

5 D. G. Creighton, «The Economic Background of the Rebellions of 1837», Canadian Journal of Economics and Political Science (CJEPS), 3 (1937): 322-34; J. Isbester, «Agriculture, Balanced Growth and Social Change in Central Canada since 1850: An Interpretation», Economic Development and Cultural Change (EDCC), 25 (1977): 673-97; N. W. Taylor, «The French Canadian Industrial Entrepreneur and his Social Environment», in M. Rioux et Y. Martin, prés., French Canadian Society, vol. 1 (Toronto, 1964). R. Durocher et P.-A. Linteau, prés., Le retard du Québec et l'infériorité économique des Canadiens français (Montréal, 1971), analysent cette famille d'interprétations.

6 P. Chaunu, "L'histoire sérielle: bilan et perspectives», Revue roumaine d'histoire, 9 (1970): 473; N. Rescher, Essays in Philosophical Analysis (Pittsburg, 1969), chap. 9 et 10.
} 
par les historiens au Canada: (1) ils ont surtout tablé sur des schémas d'interprétation impressionnistes et implicites, (2) les argumentations échafaudées ont souffert fréquemment de fautes de logique flagrantes, et (3) les canons d'admissibilité des témoignages se sont avérés clairement plus laxistes que ceux en usage dans les autres sciences humaines ou dans les cours de justice ${ }^{7}$.

Nous nous attacherons ici seulement au point (1), parce que c'est probablement la faiblesse généralisée la plus importante: celle qui, peutêtre, a entraîné les autres. Les historiens canadiens ont montré trop peu de goût pour la théorisation explicite dans leurs travaux. En conséquence, trop de prospections à l'aveuglette, servies par des méthodologies faibles et des argumentations spécieuses, ont pris des airs d'orthodoxie: ces cosmologies déficientes et floues, qu'on semble avoir voulu immuniser contre la réfutation, doivent être soumises à des analyses critiques.

\section{A. Le chromo}

Pour Chapais, Groulx, Burt, Lower, Creighton, Jones, etc. tous écrivant dans la première moitié du $20 \mathrm{e}$ siècle - l'expérience socio-économique du Bas-Canada se réduit fondamentalement au conflit entre deux philosophies, deux genres de vie: «the medieval, rural, Catholic way of life» des habitants et «the hurly-burly of the English man of business ${ }^{8}$. Les deux premiers décèlent dans ces valeurs et ces «vertus» des Canadiens français un exemple à suivre; les seconds, une faille qui a fait glisser ce peuple, par sa faute, dans une position subordonnée.

Cette dichotomie - d'ailleurs peu documentée - est en gros la pierre de touche d'à peu près tout l'appareil historiographique d'avant 1950, à quelques exceptions près. Que l'on se réfère aux mentalités,

\footnotetext{
7 Ces observations ne contestent pas la qualité du travail accompli par beaucoup de devanciers, non plus que l'existence d'une minorité d'historiens moins piégés par un tel laxisme, mais identifient certaines faiblesses assez généralisées et parfois flagrantes dans les méthodologies utilisées. Voir G. Paquet et J.-P. Wallot, «Canada 1760-1850: anamorphoses et prospectives», in R. Comeau, prés., Économie québécoise (Montréal, 1969), 255-300; S. Gagnon, Quebec and its historians, the twentieth century (Montréal, 1985); L. Dechêne, «Observations sur l'agriculture du Bas-Canada au début du XIXe siècle», communication au colloque d'histoire rurale comparée de la France de l'Ouest et du Québec, Rochefort, juillet 1982 (à paraître).

A. R. M. Lower, Colony to Nation (Toronto, 1947), 67-69; aussi idem, «Two ways of Life: The Primary Antithesis of Canadian History», CHAR (Ottawa, 1943), 5-18. «If Canadian history is to be understood at all, it is necessary first of all to be able to understand and apply to common situations these two ways of life: the static Catholic-rural, careless of well-being, not over-burdened with social responsibility, prodigal in life, welcoming many children...; and the dynamic Calvinist-commercial with its devotion to acquisition and its haunting fear of animal «robustiousness»... On the one hand stand those who are close to the soil, irrevocably committed to the land in which and by which they live [c'est nous qui soulignons]; and on the other those who simply regard mother Earth as a source of good things, who cut down its forests and tear out its minerals regardless of its future and then, if opportunity offers, rush off to pleasanter places.» (Colony to Nation (Toronto, 1947), 67-69).
} 
aux cultures, voire à la «race», les types-idéaux de l'habitant canadien et du marchand-bourgeois anglais engendrent, dans ces analyses, un dualisme culturel fondamental assez vague pour accommoder un certain genre d'explication'. Cette vision manichéenne a suscité des interprétations capricieuses et faussées d'événements aussi importants dans l'histoire du Québec que (1) la genèse de l'Acte constitutionnel de 1792, (2) la modernisation de la socio-économie québécoise au tournant du $19 \mathrm{e}$ siècle et (3) la querelle des subsides dans la première moitié du $19 \mathrm{e}$ siècle ${ }^{10}$.

Ce chromo présente une image de l'habitant qui le prive presque de cette capacité à se doter de mécanismes de survie que l'on prête pourtant même à $l^{\prime}$ 'animal ${ }^{11}$. L'habitant est considéré comme un être culturellement inférieur et démuni que Lord Durham voudra délivrer de sa misère à la fin des années $1830^{12}$. Pas question de conjecturer qu'il puisse avoir pensé et cherché à réaliser des stratégies gagnantes sur les plans constitutionnel, socio-économique ou politique. Analphabétisme, ignorance, conservatisme, catholicisme, traditionalisme, etc. sont devenus des flétrissures interchangeables, voire cumulatives.

Ce chromo laisse également son empreinte sur beaucoup de travaux d'historiens québécois et canadiens de la seconde moitié du 20e siècle. Plus près de nous, Maurice Séguin, W. H. Parker, Albert Faucher et Fernand Ouellet ont, jusqu'à un certain point, construit des variations autour de cette dichotomie, quoique avec un bonheur inégal et des niveaux différents de simplification. Le discours s'est raffiné, la discussion a revêtu un ton plus technique et le dossier documentaire s'est enrichi; mais pour l'essentiel, la weltanschauung a peu bougé ${ }^{13}$.

9 Ce dualisme culturel est aussi assez vague pour accommoder toutes les extravagances: on en arrive même à attribuer toutes les différences observées entre les deux groupes au principe de classification. C'est le vieux péché logique - ignoratio elenchi. (P. Coffey, The Science of Logic (New York, 1912), 2: 301). Pour une critique de cette vision, voir S.Courville, "L'habitant canadien dans la première moitié du 19e siècle: survie ou survivance?», miméo, 1985.

${ }_{10}$ V.g. voir $\mathrm{P}$. Tousignant, La genèse et l'avènement de la Constitution de 1791 , thèse de Ph.D. man., U. de M., 1971; G. Paquet et J.-P. Wallot, Patronage et pouvoir dans le BasCanada, 1793-1812 (Montréal, 1973).

11 Les humains ne sont pas les seuls êtres susceptibles de créer des institutions ou des conventions. M. Fredlund a montré comment des animaux sont parfaitement capables de créer des systèmes de droits de propriété territoriale et de distribution de l'espace qui ressemblent étrangement aux nôtres et fonctionnent sur le même principe. («Wolves, Chimps and Demsetz», Economic Inquiry, 45 (1976): 279-291). L'historiographie conventionnelle en arrive presque à refuser de reconnaître que l'habitant puisse faire aussi bien!

12 C'est ce chromo durable et ancien qui sert à Lord Durham dans la seconde partie de son fameux rapport.

13 Pour des illustrations, voir G. Paquet et J.-P. Wallot, «Groupes sociaux et pouvoir: le cas canadien au tournant du 19e siècle», RHAF, 27,4 (mars 1974): 509-564. F. Ouellet utilise même l'expression «eternal looser» (sic) pour caractériser l'habitant. (F. Ouellet, Éléments d' histoire sociale du Bas-Canada (Montréal, 1972), 214; aussi idem, «Le mythe de l'habitant sensible au marché», Recherches sociographiques, 17 (1976): 115-132). J. McCallum a réfuté plusieurs explications fondées sur cette dichotomie (Unequal Beginnings (Toronto, 1980), chap. 9). 


\section{$B$. Le modèle}

L'image de rechange que nous proposons de l'habitant entend l'exorciser de cette ignorance comme de ce conservatisme congénital et l'extraire de ce tissu social déterministe, pour le considérer simplement comme un être rationnel, armé comme tous les autres agents économiques, d'une rationalité limitée (bounded rationality) et soumis, dans son processus de prise de décision, aux contraintes normales imposées par les coûts de décision et de transaction ainsi que par les circonstances du milieu ambiant. Comme les contraintes auxquelles l'habitant est confronté peuvent être différentes de celles auxquelles font face ses compatriotes anglophones, ses comportements pourront s'avérer autres: non pas par conservatisme inné, mais parce que le contexte différent commande une stratégie différenciée. Les écarts dans les coûts de l'information et dans les coûts d'accès au capital pousseront en particulier l'habitant à choisir des stratégies économiques spécifiques ${ }^{14}$.

L'héritage culturel - coutumes, lois et règles particulières du jeu social - existe; mais la plasticité de ces institutions se révèle plus grande qu'on ne le soupçonne. De plus, la dynamique adaptation/adoption, qui les conforme, prend sa source dans les choix d'agents économiques rationnels et non pas dans des réactions entièrement programmées par la culture ${ }^{15}$. L'important, dans cette image de rechange, c'est qu'elle se fonde sur un modèle d'habitant pro-actif opérant de manière stratégique dans un milieu qui l'oblige à manoeuvrer différemment selon les «circonstances». Elle récuse le fatalisme et le déterminisme de l'«eternal looser» (sic). De plus, de cette image de rechange, ne coulent plus aussi aisément toutes les conséquences acceptées trop cavalière-

14 C'est la position que nous avons défendue depuis 1972 (G. Paquet et J.-P. Wallot, «Crise agricole et tensions socio-ethniques dans le Bas-Canada, 1802-1812)». Il ne s'agit pas de nier l'existence d'un éthos qui donne une plus grande probabilité à certaines réactions ou à certains mécanismes d'adaptation, d'un éthos qui adopte aussi certains agents et leur donne une plus grande probabilité de survie et de succès. C'est la dynamique adaptation/adoption. Mais l'éthos résulte lui-même des activités et des choix de la multitude des membres d'une communauté et sa conformation dépend en grande partie de son utilité, de sa «fonctionalité». Il se peut donc que l'on doive considérer la culture non seulement comme une contrainte, mais encore et surtout comme un construit, non pas comme un déterminisme, mais comme une technologie sociale, comme un mécanisme de survie. Pour une analyse de la dynamique adaptation/adoption, voir A. A. Alchian, «Uncertainty, Evolution and Economic Theory», The Journal of Political Economy 58 (1950): 211-221; voir aussi G. Paquet et J.-P. Wallot, «Nouvelle-France/Québec/Canadas: a World of Limited Identities», Working Paper 83-56 (Faculté d'administration, Université d'Ottawa, 1983), $73 \mathrm{p}$.

15 Voir G. Paquet et J.-P. Wallot, «Crise agricole et tensions socio-ethniques dans le BasCanada, 1802-1812». Cette image de l'habitant rationnel semble coller bien mieux à la réalité concrète décrite par les observateurs/voyageurs du temps comme Lambert (Travels Through Lower Canada [...], (Londres, 1810, 3 vol.), cité en épigraphe, et qui présentent l'habitant comme un agent économique astucieux. Cela ne veut pas dire que les institutions que l'on observe dans le Bas-Canada ont été nécessairement choisies et voulues par l'habitant: les institutions sont le plus souvent le résultat non voulu et non prévu de l'action des hommes; mais on peut comprendre quelles formes prendront les patterns d'organisation et d'accumulation à partir des forces harnachées (délibérement ou non) par l'action rationnelle des agents économiques impliqués. Voir T. C. Schelling, Micromotives and Macrobehavior (New York, 1978). 
ment par l'historiographie: morcellement du sol, techniques agricoles désuètes, crise agricole du début $19 \mathrm{e}$ siècle, infériorité économique inévitable. Tout est remis en question.

\section{La stratégie économique des habitants}

De nombreux travaux récents ont discrédité l'hypothèse d'une crise agricole et d'une chute radicale des rendements agricoles dès le début du $19 \mathrm{e}$ siècle, de même que la prétention que les habitants utilisaient des techniques déraisonnables ${ }^{16}$. On a aussi commencé à déchiffrer derrière certaines conduites surprenantes de l'habitant les contours d'une stratégie économique claire qui privilégie le capital foncier et le patrimoine dans son programme économique. Loin d'incarner un signe de rejet du capitalisme, un conservatisme inné, une mentalité d'Ancien Régime ou un repli dysfonctionnel sur le secteur économique traditionnel, cet intérêt de l'habitant pour la terre correspondrait à une stratégie d'accumulation tout à fait rationnelle. L'historiographie canadienne a eu peut-être le tort de se laisser trop influencer par certains historiens anglais et américains pour qui commerce et industrie sont synonymes de dynamisme, et terre, de conservatisme. Linteau et Robert ont montré qu'il n'en était rien pour Montréal ${ }^{17}$. Or, se pourrait-il qu'en campagne également, l'investissement dans la terre constitue un investissement fort rentable et que ce secteur soit celui dans lequel l'habitant canadien puisse opérer avec le minimum de désavantages par rapport à ses concurrents britanniques entre 1760 et 1850 ?

Si cette hypothèse, qui élargit encore celle déjà générale énoncée par Linteau et Robert, est recevable, on devrait pouvoir constater que les habitants ont développé des règles et pratiques susceptibles tant de préserver l'intégrité des patrimoines existants que de constituer des patrimoines plus vastes. Le choix du capital foncier par opposition au capital commercial ou industriel représenterait alors un ajustement rationnel aux différentiels de coûts d'information et de finance qui affligent les habitants canadiens avant le développement de systèmes financiers indépendants des réseaux de parenté et libres des liens avec les grands marchands métropolitains ${ }^{18}$. Plus tard, c'est probablement,

16 G. Paquet et J.-P. Wallot, «Crise agricole [...]»; R. M. McInnis, «A Reconsideration of the State of Agriculture in Lower Canada in the First Half of the Nineteenth Century», in D. H. Akenson, prés., Canadian Papers in Rural History (Gananoque, 1982), 9-49; J. McCallum, Unequal Beginnings (Toronto, 1980), chap. 3; S. Courville, «La crise agricole du Bas-Canada: éléments d'une réflexion géographique», Cahiers de géographie du Québec, 24 (1980): 193-224; L. Dechêne, «Observations sur l'agriculture du Bas-Canada [...]».

17 P.-A. Linteau et J.-C. Robert, «Propriété foncière et société à Montréal: une hypothèse», $R H A F, 28,1$ (juin 1974): 45-65.

18 Sur l'évolution du système financier, voir G. Paquet et J.-P. Wallot, «Le système financier bas-canadien au tournant du XIXe siècle», L'Actualité économique, 59 (1983): s. 4. Linteau et Robert parlent d'un type d'avantage comparatif informationnel et politique des francophones sur les marchés fonciers, vers 1825, à Montréal. 
comme le suggèrent Linteau et Robert, à cause de la qualité plus grande de l'information dont ils disposent sur le marché foncier et des influences qu'ils peuvent exercer sur les administrations locales, que les Canadiens continueront de détenir un avantage comparatif dans ce secteur.

Ce serait donc la configuration de l'espace des possibilités effectives de l'habitant qui l'aurait acculé à choisir cette stratégie plutôt que des différences de mentalité ou un degré d'aversion pour le risque plus fort chez les habitants que chez leurs compatriotes britanniques ${ }^{19}$. En fait, le choix du capital foncier semble avoir été fait rationnellement dans la direction de la moindre résistance.

\section{A. Pratiques pour maintenir l'intégrité du patrimoine}

La Coutume de Paris, même amendée par divers édits et ordonnances, s'appliquait au Québec depuis le 17e siècle et imposait, croiton, la répartition égale du patrimoine de la communauté entre tous les enfants (fils et filles); elle aurait réglé dans les menus détails la dévolution des héritages, en tenant compte des propres, des douaires, des préciputs, etc. En 1971, Yves Zoltvany notait la rigueur de la Coutume et la reliait au «conservatisme» de la société québécoise. Il parlait d'inaptitude au capitalisme et de morcellement ruineux des patrimoines $^{20}$.

$\mathrm{Au}$ cours des dernières années, la recherche a montré que cette vision était tronquée. Ainsi, en 1974, Louise Dechêne détectait des dérogations nombreuses et systématiques à la Coutume de Paris au Québec: dérogations non seulement tolérées, mais même officialisées par des actes notariés (par exemple, fusion des propres dans la communauté, dons réels entre conjoints vifs, répartition habile de l'héritage de façon à avantager un héritier principal, etc., sans compter le règlement de l'héritage avant le décès par donation, avances d'hoirie et dévolution de l'exploitation à l'un des enfants par consensus familial ou rachat partiel). La plupart du temps, on ne contredit pas la Coutume de Paris, mais on assure par toute une série d'actions parallèles, la transmission aussi intégrale que possible du patrimoine ${ }^{21}$.

Ce même souci du maintien de l'intégrité du patrimoine se retrouve d'ailleurs dans les stratégies matrimoniales, comme l'ont montré Jacques

19 Pour une explication des différences entre ces deux approches mettant l'accent sur les aspects subjectifs et objectifs de la situation respectivement, voir l'appendice au présent article; aussi I. Adelman, «Social and Economic Development at the Micro Level - A Tentative Hypothesis», in E. B. Ayal, prés., Micro Aspects of Development (New York, 1973), 3-13. 384.

Y. Zoltvany, «Esquisse de la Coutume de Paris», RHAF, 25,3 (décembre 1971): 365 1974) L. Dechêne, Habitants et marchands de Montréal au XVIIe siècle (Montréal et Paris, 
Mathieu et son équipe pour les $17 \mathrm{e}$ et $18 \mathrm{e}$ siècles $^{22}$. Louis Michel, dans une étude longitudinale des paroisses de Varennes et de Verchères aux $18 \mathrm{e}$ et $19 \mathrm{e}$ siècles, souligne que la famille assure la transmission du patrimoine principal, sans morcellement, grâce à des ententes familiales et notamment la donation, l'acte de vente fictif ou partiel, l'avance d'hoirie, etc. On voit même se reproduire de cette façon toute une hiérarchie sociale: les gros habitants établissent tous leurs enfants sur des terres de bonne dimension alors que les plus pauvres n'y parviennent pas et doivent les envoyer ailleurs. En fait, établir les enfants deviendrait la grande idée-force derrière cette stratégie d'accumulation ou de conservation du patrimoine foncier ${ }^{23}$.

L'examen de terriers confirme que le morcellement des terres ne se produit pas: les démembrements, lorsqu'ils surviennent, sont généralement suivis d'un remembrement rapide ${ }^{24}$. Par ailleurs, Serge Courville a montré qu'il n'y a pas de morcellement du patrimoine, mais donation ou rachat partiel à chaque génération. Si certains historiens ont conclu trop vite à un morcellement catastrophique du patrimoine foncier dans les années 1820-30, c'est peut-être qu'en utilisant les recensements et en calculant la superficie des terres par habitant, ils ont confondu villageois et habitants, ce qui déprime considérablement les moyennes $^{25}$. Les exploitations, en fait, demeurent de taille importante ${ }^{26}$.

22 A leur avis, les familles sont plus prodigues de leurs filles que de leurs terres: mariages entre plusieurs filles d'une même famille et plusieurs frères d'une seconde pour renforcer les liens de parenté et les avoirs patrimoniaux (J. Mathieu, «Les alliances matrimoniales exogames dans le gouvernement de Québec, 1700-1760», RHAF, 35,1 (juin 1981): 3-32; idem, «Mobilité et mariage dans le gouvernement de Québec au XVIIIe siècle», communication au colloque de Rochefort, 1982 (à paraître). L. Lavallée arrive à la même conclusion («La transmission du patrimoine dans la seigneurie de Laprairie (1667-1760)», communication au colloque de Rochefort, 1982 (à paraître).

23 On trouvera des éléments de cette interprétation encore largement inédite dans L. Michel, «Un marchand rural en Nouvelle-France: François-Augustin Bailly de Messein, 1709-1771», RHAF, 33,2 (septembre 1979): 215-262; idem, «Le livre de comptes de Gaspard Massue, marchand à Varennes, 1784-1792», Histoire sociale, 13 (1980): 369-398; idem, "Varennes et Verchères des origines au milieu du XIXe siècle. État d'une enquête», communication au colloque de Rochefort, 1982 (à paraître). Voir aussi G. Bouchard, «Sur l'historiographie des campagnes et des régions du Québec aux XIXe et XXe siècles: nouvelles propositions", in Histoire sociale, sensibilités collectives et mentalités. Mélanges Robert Mondrou (Paris, 1985), 561-571. Une telle stratégie n'est pas exclusive aux habitants (des seigneurs et des marchands, par exemple, y ont recours) et n'exclut pas d'autres motivations: enrichissement, statut social, etc.

${ }_{24}$ V.g. voir, aux ANQ-Q, le terrier de la seigneurie de Lauzon (1824) qui retrace l'évolution de toutes les propriétés sur cette seigneurie.

${ }_{25} \mathrm{~S}$. Courville, L'habitant canadien et le système seigneurial, 1627-1854, thèse de Ph.D. man., Université de Montréal, 1979; idem, «La crise agricole au Bas-Canada»; idem «Esquisse de la croissance des villages au Québec: le cas de l'aire seigneuriale entre 1760 et 1854 », $C G Q$ 28 (1984): 9-46; idem, «Villages et agriculture dans les seigneuries du Bas-Canada: éléments pour une réinterprétation du développement rural québécois dans la première moitié du $19 \mathrm{e}$ siècle», miméo, juin 1984, 38 p. Courville souligne l'extrême difficulté de comparer les mêmes paroisses dans le temps (nombreuses divisions) et les populations (croissance très rapide des villages entre 1800 et 1850 , absence de distinction entre habitants véritables et les autres, etc.). Il faut littéralement reconstruire les statistiques à partir des recensements nominatifs, des cartes militaires, des aveux et dénombrements, des études de Bouchette $(1815,1832)$, des cadastres abrégés des seigneuries (1863), des plans de villages, des histoires et monographies locales, etc. En 1831, plus de 40000 personnes vivraient dans les villages, soit autant que la population des villes de Québec, 


\section{B. Différenciation sociale et capital foncier}

La pénétration du marché dans le Bas-Canada a fait surgir une différenciation sociale qui se démarque clairement dans la structuration différente de la richesse mobilière ${ }^{27}$ et des avoirs immobiliers. Une «gentilité» d'habitants émerge, dotée de patrimoines imposants, alors qu'un nombre croissant d'habitants se rabattent sur les villes et sur les villages qui se multiplient entre 1790 et $1830^{28}$. Ce procès de diversification se déploie plus lentement dans la propriété immobilière, moins sensible aux soubresauts conjoncturels que la richesse mobilière ${ }^{29}$. Par contre, la discontinuité enclenchée dans le secteur foncier est plus fondamentale et permanente: la structure des stocks de richesse immobilière, dans une économie où les habitants forment de $75 \%$ à $80 \%$ de la population, fonde à plus long terme la structure de la richesse tout court et de la répartition des revenus ${ }^{30}$. Même l'éthos et les règles du jeu se transformeront en conséquence.

\section{Esquisse de la stratégie foncière de l'habitant}

L'habitant vit en régime incertain et fait face à des contraintes quant à la quantité de capital qu'il peut mobiliser ${ }^{31}$. Dans ces circonstances, faut-il se surprendre s'il oriente prudemment son activité vers le secteur qu'il connaît le mieux, où l'incertitude s'avère relativement moindre et qui requiert une quantité moindre de capital financier relativement rare. Il va aussi utiliser des techniques de production qui économisent ce capital rare. On a conclu trop vite au conservatisme ou à l'aversion pour le risque; mais, comme J. Roumasset l'a bien montré,

Trois-Rivières et Montréal. D'où la difficulté d'estimer sommairement les superficies occupées et cultivées par habitant. Aussi, A. R. Greer, Habitants of the Lower Richelieu: Rural Society in the Three Quebec Parishes, 1740-1840, thèse de Ph. D. man., Université York, 1980.

26 Selon M. Séguin, par exemple, l'exploitation-type, autour de 90 arpents en 1765, demeurerait encore aux environs de 80 arpents après 1850 (La nation «canadienne» et l'agriculture (1760-1850) (Trois-Rivières, 1970), 172-178, 220).

27 Sur cette question, voir G. Paquet et J.-P. Wallot, «Les inventaires après décès à Montréal au tournant du XIXe siècle: préliminaires à une analyse», RHAF, 30,2 (septembre 1976): 163-221; les articles de Paquet et Wallot, de G. Bervin, J.-P. Hardy, T. Ruddell, C. Dessureault et L. Gadoury dans Bulletin d' histoire de la culture matérielle (BHCM), 17 (1983).

25 .

28 Sur les villages, voir $S$. Courville, «Esquisse du développement villageois [...]» et note

29 Nous avons clairement montré en 1976 comment les discours de la production et de la consommation - des flux - avaient été transformés à Montréal entre la fin du 18e et le début du $19 \mathrm{e}$ siècle. («Les inventaires après décès $[\ldots]$ », $195 \mathrm{ss}$ ).

30 On n'a pas assez insisté dans les études spécialisées sur les rapports stables entre les flux de consommation et les stocks de la richesse (voir R. W. Clower et M. B. Johnson, "Income, Wealth, and the Theory of Consumption», in J. N. Wolfe, prés., Value, Capital and Growth (Edinburgh, 1968), 87 ss; idem, «Stock-Flow Analysis», International Encyclopedia of Social Sciences, 15 (1968): 273-277).

${ }_{31}$ Voir G. Paquet et J.-P. Wallot, «Crise agricole [...]», 201-216. 
on assimile trop facilement à une aversion pour le risque ce qui peut découler tout simplement des imperfections dans le marché du capital ${ }^{32}$.

La rationalité du comportement de l'habitant paraît nette: vivant dans un monde où l'information qui lui arrive à propos de la demande effective et des prix de ses produits est incertaine et souvent médiatisée par des groupes concurrents, largement exclu des grands réseaux financiers, l'habitant vise à améliorer son sort et à installer ses enfants. Pour ce faire, il essaie moins de maximiser ses taux de profit à court terme que de maximiser sa richesse terminale transférable à ses enfants sous la contrainte d'une mise de fonds originelle limitée par un manque d'accès au marché du capital. Trop d'auteurs n'ont pas compris qu'une stratégie rationnelle ne se réduit pas nécessairement à la maximisation du taux de profit à court terme ${ }^{33}$. Par rapport à l'optimum prévisible dans un monde simple, certain et en concurrence parfaite, l'habitant semble de prime abord avoir adopté une stratégie de sous-optimisation prudente (cautious sub-optimizing). Or, en régime économique où règnent la complexité, l'incertitude et l'imperfection du marché du capital, cette stratégie est justement préférable et produit des performances meilleures que celles de l'optimisation simple ${ }^{34}$.

Bref, l'habitant oriente son activité vers un secteur (1) où l'incertitude effective est relativement moindre, et (2) où le besoin en capital financier est relativement moins important; et dans le secteur élu, (3) il opte pour des techniques qui requièrent relativement moins de capital et, (4) à cause justement du manque de capital, il ne pousse pas la production, comme le suggérerait un processus d'optimisation simple,

32 Selon J. A. Roumasset, on peut expliquer des comportements de fermiers ressemblant à de l'aversion pour le risque grâce à un modèle de maximisation du profit anticipé qui tient compte des imperfections dans le marché du capital. («Risk Aversion, Indirect Utility Functions, and Market Failure», in J. A. Roumasset, J. M. Boussard et I. Singh, éd., Risk, Uncertainty, and Agricultural Development (New York, 1979), chap. 6, s. 2.3).

${ }_{33}$ L'objectif du fermier capitaliste est de maximiser sa richesse terminale sous la contrainte de sa mise de fonds initiale. C'est équivalent à la maximisation du profit total pour la n-ième période, étant donné la contrainte sur le capital financier; mais ce n'est pas équivalent à la maximisation du taux de profit. Chez les spécialistes, on a décomposé le problème en deux étapes: (1) la maximisation du revenu total pour une mise de fonds donnée: elle implique une allocation optimale des ressources disponibles selon la contribution marginale des différents inputs. Or comme la terre abonde (du moins jusque vers 1820), alors que le capital et le travail sont rares, l'habitant veut maximiser le revenu total et donc minimiser le gaspillage en utilisant peu de travail et de capital par unité de terre. C'est ce qui explique la logique du «soil mining» - expression un peu inexacte pour le Québec du 19e siècle - qu'on observe tant au Canada qu'aux Etats-Unis dans l'agriculture du $18 \mathrm{e}$ et du début du 19e siècle. (W. C. Scoville, «Did Colonial Farmers «Waste» Our Land?», The Southern Economic Journal, 20 (1953): 178-181; G. Paquet et J.-P. Wallot, «La crise agricole [...], 218-221). (2) La maximisation de la richesse terminale à partir du choix de la mise de fonds initiale: quand l'accès au capital est limité, ce choix se trouve lui-même contraint. Il se peut alors que la richesse terminale soit bien moindre que si la mise de fonds initiale avait été plus grande. Le rationnement effectif de capital réduira la richesse terminale, mais ceci ne remet pas en question la rationalité du fermier. Voir J. M. Currie, The Economic Theory of Agricultural Land Tenure (Cambridge, 1981), chap. 4.

34 R. H. Day, «Cautious Suboptimizing», in J. A. Roumasset, J. M. Boussard et I. Singh, éd., op. cit., chap. 7 . 
jusqu'au point où le produit d'une unité de capital additionnelle égalerait le coût supplémentaire en capital.

La terre devient l'actif privilégié; et comme il s'agit d'un actif qui va en s'appréciant, surtout dans la région de Québec où la quantité de bonne terre disponible a toujours été plus rare, des gains spéculatifs importants sont possibles. On devrait donc s'attendre à trouver plus tôt à Québec les signes d'une stratégie d'accaparement des terres dans les campagnes par les habitants, ainsi que d'une multiplication plus précoce de paysans sans terre devant gagner leur vie à la ville ou au village. De plus, si l'expérience bas-canadienne se rapproche de l'expérience américaine, on doit pouvoir conjecturer une corrélation positive entre le nombre d'enfants, d'une part, et la richesse terminale accumulée, d'autre part. En effet, les enfants sont à la fois un stimulant à l'accumulation de la terre nécessaire pour leur établissement, mais aussi un capital humain important susceptible de faciliter et d'accélérer la stratégie d'accumulation ${ }^{35}$.

\section{Nature et origine des données}

Pour vérifier ces conjectures quant à l'existence et à la nature du procès d'accumulation du capital foncier par les habitants, il faudrait disposer de données précises et périodiques faisant le point sur les stocks de richesse détenus par les individus. Malheureusement, il y a absence de recensement fiable et complet entre 1790 et 1831, voire peut-être $1851^{36}$. De plus, les moyennes qu'on a extraites de ces recensements ne tiennent pas souvent compte des flottements dans les définitions socio-professionnelles, dans les frontières paroissiales, dans la structure des occupations et la localisation géographique des individus recensés: par exemple, certains «habitants» sont-ils de vrais agriculteurs, des rentiers ou des artisans? Vivent-ils sur des fermes, dans des faubourgs ou des villages? Comment établir alors des moyennes fiables?

A défaut de pouvoir tabler sur des données solides et comparables pour la période 1792-1835 à partir de recensements, il nous a fallu recourir à une autre source qui permet d'ajouter à l'analyse des flux, fondée sur des séries d'indices divers (prix, salaires et revenus, etc.), une étude indispensable des stocks, nécessaire à une estimation de l'importance des transformations dans la taille et la structure des actifs survenues dans l'économie.

35 W. H. Newell, «The Wealth of Testators and its Distribution: Butler County, Ohio, 1803-1865», in J. D. Smith, éd., Modeling the Distribution and Intergenerational Transmission of Wealth (Chicago, 1980), chap. 2; G. S. Becker, «An Economic Analysis of Fertility» in G. S. Becker, The Economic Approach to Human Behavior (Chicago, 1976), chap. 9; A. Barthez, Famille, travail et agriculture (Paris, 1982).

36 Voir les mises en garde de S. Courville («Villages et agriculture [...]»; «L'habitant canadien dans la première moitié du 19e siècle»), R. M. McInnis («Some Pitfalls in the 18511852 Census of Agriculture in Lower Canada», Histoire sociale, 14 (1981): 220 ss) et H. Charbonneau (La population canadienne. Études rétrospectives (Montréal, 1973), introduction). 


\section{A. Les inventaires après décès}

Nous avons exposé ailleurs les lignes maîtresses de notre enquête sur les utilisations des inventaires après décès en histoire économique et sociale du Bas-Canada ${ }^{37}$. Rappelons que l'inventaire après décès dénombre, article par article, les biens, titres, papiers, espèces et immeubles d'un individu ou de sa communauté, généralement à la suite d'un décès. Nos articles de 1976 et de 1983 s'attardaient à décrire les nombreuses difficultés ${ }^{38}$ que pose l'utilisation de ce matériau très précieux par ailleurs.

Malgré ces difficultés, la richesse de cette source, utilisée massivement sous forme de séries, reste incontestable et son bon usage offre une vue d'ensemble permettant de saisir les ménages et les micro-communautés dans les bases concrètes de leur vie: «les objets, les instances matérielles qui décrivent un cadre de vie indentifiant un comportement et le support économique qui les rassemblent en patrimoine... ${ }^{39} \mathrm{La}$ constitution d'une banque de données à partir de plus de 1000 inventaires après décès répartis entre les régions de Québec et de Montréal, dont environ 950 pour les sous-périodes 1792-1796, 1807-1812, 18201825 et 1830-1835, a permis de baliser l'évolution socio-économique du Bas-Canada.

En ce qui a trait à la région du Québec, l'objet du présent article, l'échantillon global dépasse 425 observations et couvre tant la ville de Québec proprement dite (115) que la rive nord (202) et la rive sud (109). Malgré des recherches intensives, les données disponibles comportent des lacunes flagrantes pour certaines occupations, d'où la difficulté de reconstruire un «portrait» socio-économique complet de cette région. Toutefois, en ce qui concerne les habitants, sur lesquels porte le présent article, la taille de l'échantillon est suffisante pour la rive nord (192 observations - 55, 62, 42 et 33 respectivement pour les quatre souspériodes) et pour la rive sud (102 observations - 29, 37, 17, 19 respectivement pour les quatre sous-périodes) tout autant que pour chacune des tranches chronologiques.

Il nous a fallu composer avec certaines imprécisions dans les inventaires et prendre certaines décisions lorsqu'une fourchette d'interprétations était possible ou lorsque quelques observations pouvaient engendrer des distortions majeures dans les moyennes générales.

37 G. Paquet et J.-P. Wallot, «Les inventaires après décès [...]»; idem, «Structures sociales et niveaux de richesse [...]»; idem et al., «Culture matérielle et société au Québec, 1792-1835», BHCM, 17 (1983): 9-23.

38 V.g. exactitude et sincérité variables des notaires, des témoins et des priseurs; omissions et imprécisions dans l'énoncé et les mesures des stocks de consommation ainsi que des immeubles, etc. (ibid.; J.-P. Hardy, «Niveaux de richesse et intérieurs domestiques [...]», BHCM, 17 (1983): 63-94).

D. Roche, Le peuple de Paris (Paris, 1981), 59. Outre les études déjà mentionnées, voir L. Gadoury, «Les stocks des habitants dans les inventaires après décès», $B H C M, 17$ (1983): 139 147. 
(1) Les inventaires ne fournissent que rarement les mesures des maisons et des bâtiments, bien qu'ils nous renseignent davantage sur le nombre d'étages et le matériau principal de leur construction. Il précise occasionnellement le nombre d'arpents ensemencés et cultivés, par rapport à la superficie globale. Parfois des notaires se contentent d'évoquer très vaguement la superficie de certaines terres: «trois arpents de front jusqu'au bout du chemin» ou «jusqu'à la rivière» ou «jusqu'au chemin du roi» ou, pis encore, «jusqu'à la limite»! Dans tous les cas, nous avons adopté une approche conservatrice, de sorte que les chiffres compulsés et les moyennes qui en sont déduites, doivent être considérés comme des minima.

(2) Il a fallu exclure des calculs les quelques propriétaires - des urbains - de plus de 2000 arpents (généralement dans les cantons), car ils faussaient gravement les moyennes et créaient une distorsion importante dans la présentation des exploitations réelles. Cependant, nous avons noté la présence de ces propriétaires chaque fois, ainsi que la superficie totale de leurs possessions ${ }^{40}$.

(3) Nous avons pu estimer assez précisément la superficie de terres situées sur l'île d'Orléans et dont la profondeur n'est pas établie dans l'inventaire. En effet, les inventaires plus précis établissent la profondeur des terres, dans les mêmes rangs, à l'intérieur d'une fourchette de 55 à 65 arpents. Nous avons opté pour la mesure minimale (55). Afin de nous assurer que le lecteur puisse toujours faire abstraction de ces évaluations imparfaites, nous avons retenu pour la rive nord deux séries de calculs: l'hypothèse $a$, qui ne comprend que les minima certains et exclut les terres dont la superficie est inconnue ainsi que les quelques trop gros propriétaires; l'hypothèse $b$, basée sur les mêmes calculs, mais qui leur ajoute certaines approximations plausibles pour un certain nombre de terres à superficie inconnue sur l'île d'Orléans.

(4) Finalement, lorsque les superficies sont exprimées en acres, ceux-ci ont été convertis en arpents au taux de 1.18 arpents à l'acre. Pour ce qui est des lots dans les cantons, il nous est arrivé à une reprise (en 1807-1812) de postuler que le lot comportait la superficie réglementaire, soit 200 acres par lot numérotét ${ }^{41}$.

40 S'il avait fallu tenir compte des immenses propriétés de cinq marchands ou membres des professions libérales dans les villes, propriétés qui pouvaient être des seigneuries ou, plus souvent, de vastes étendues de terres incultes dans les cantons, les moyennes auraient été gravement faussées en ce qui a trait à la situation véritable de la quasi-totalité de la population. Ainsi, en 17921796, la grande moyenne passerait de 74 arpents à 540 arpents, en 1807-1812, de 115 ou 122 arpents à 652 arpents, etc. Ont donc été exclus des calculs en ce qui a trait aux superficies, les cas suivants: 1792-1796, Ville de Québec, 1 marchand, 37397 arpents; 1807-1812, Ville de Québec, 2 marchands, 9098 et 5192 arpents, et 1 membre des professions libérales, 59472 arpents; 1830-1835, Ville de Québec, 1 marchand, 7062 arpents.

${ }^{41}$ Un arpent compte 10 perches, et la perche, 18 pieds. Dans l'agrégation de certaines parcelles de faible superficie, nous avons ignoré les mesures en-deçà de 9 pieds (dans le calcul des perches). Voir R. M. McInnis, «Some Pitfalls in the 1851-1852 Census [...]». 


\section{B. Un échantillon représentatif}

Reste à voir si cet échantillon d'inventaires après décès choisis au hasard, complété par des efforts ponctuels pour retracer des inventaires dans les catégories socio-professionnelles sous-représentées, est suffisamment représentatif de la réalité socio-économique bas-canadienne. Cette question a été débattue en détail dans des travaux antérieurs. Notre examen critique, auquel s'ajoutent des tests ultérieurs par divers chercheurs sur des parties de l'échantillon et sur les données qui en sont tirées, nous a convaincus que notre échantillon est représentatif. Les travaux de Y. Morin (paroisse Notre-Dame-de-Québec), de L. Déry et R. Lessard (ville de Québec et faubourgs), de C. Dessureault (seigneurie de St-Hyacinthe), et de nombreuses thèses confirment que notre grille d'échantillon représente bien la structure occupationnelle de la population, de leur localisation géographique et de la répartition de leurs biens. On peut aussi faire bon usage de nombreuses études étrangères, telles celles des Daumard, Meyer, Labarra, etc., pour étayer nos présomptions sur la représentativité des inventaires après décès en général comme indicateur social ${ }^{42}$.

Malgré la prudence de nos conjectures, le luxe de détails dans l'explication de la construction de notre échantillon, la longueur et la complexité des analyses dont les résultats démontrent des variations parfois importantes, Fernand Ouellet a commenté de façon presque diffamatoire nos «résultats extraordinairement et universellement positifs» et a nié capricieusement le caractère représentatif de notre échantillon ${ }^{43}$.

42 Un travail inédit de L. Déry et de R. Lessard («Esquisse de la situation socio-matérielle des forgerons, menuisiers et charpentiers de Québec, 1792-1835», miméo, Musée de l'Homme, été 1984) démontre que notre échantillon est très représentatif de la proportion et de la localisation des groupes sociaux retenus. Pour sa part, Y. Morin a illustré la représentativité des inventaires après décès à Québec pour la période qui nous intéresse («La représentativité de l'inventaire après décès - l'étude d'un cas: Québec au début du XIXe siècle», RHAF, 34,4 (mars 1981): 515-533). Par ailleurs, des thèses qui ont fait une utilisation plus exhaustive des inventaires après décès pour une période et une région plus restreintes, confirment cette représentativité et les résultats de nos premières analyses (par exemple, G. Bervin, Les marchands-négociants à Québec au début du XIXe siècle [...], thèse de M.A. man., Université de Montréal, 1981; J. Denys, L'habitant de Laprairie (1790-1835) et la crise agricole, thèse de M.A. man., Université de Montréal, 1980; $\mathrm{G}$. Desroches, Les niveaux de fortune de quelques groupes socio-professionnels de la région de Chambly (1815-1835), thèse de M.A., man., Université de Montréal, 1979). Il en est de même des travaux actuels de C. Dessureault sur la seigneurie de Saint-Hyacinthe à partir d'un vaste ensemble d'actes notariés et d'autres sources disponibles. Il démontre, entre autres, qu'à SaintHyacinthe, la représentativité des inventaires dépasse $55 \%$ des habitants adultes morts au-dessus de 20 ans. Il n'y a aucune raison de croire qu'il n'en est pas ainsi dans la plupart des campagnes québécoises.

${ }_{43}$ A son avis, nos chiffres comporteraient des écarts «aberrants» par rapport à ceux qu'il a lui-même inférés de recensements (v.g. les superficies moyennes des terres «dans» les villes, les moyennes d'animaux); nous n'aurions pas démontré la représentativité des inventaires ni leur pertinence comme indice; notre méthodologie ne serait pas claire et notre échantillon serait ainsi construit qu'il conduirait inévitablement à des résultats positifs pour nos hypothèses. Enfin, nous comparerions une période de «basse conjoncture» (1792-1796) et une autre de «haute conjoncture» (1807-1812). (F.Ouellet, "Les classes dominantes au Québec, 1760-1840. Bilan historiographique», RHAF, 38,2 (automne 1984): 232-234). Si ce détracteur avait pris la peine de lire nos 
Les lecteurs plus objectifs se reporteront à nos longs exposés de la méthode de construction de notre échantillon ${ }^{44}$. Il convient peut-être d'en rappeler une ou deux dimensions. Ainsi, les inventaires ont été choisis au hasard, sans écarter ni les riches ni les pauvres, mais en éliminant (lorsque l'information existait) les personnes trop jeunes (dans la vingtaine) ou trop vieilles (au-delà de 60-65 ans). A vrai dire, sauf pour les groupes nombreux comme les habitants à l'extérieur des villes (représentativité d'au moins 15\%), l'échantillon comprend de $50 \%$ à $100 \%$ de tous les inventaires trouvés. On peut consulter les documents de travail à la fois aux Archives publiques du Canada et au Musée de l'Homme à Ottawa.

Les inventaires après décès enregistrent des stocks de richesse et donc sont moins sensibles aux soubresauts conjoncturels, d'une année à l'autre, que ne pourraient l'être les flux de revenus et de consommation. On notera que la tendance des années 1807-1812, pour plus accélérée qu'elle paraisse, trouve des prolongements inégaux dans les années 1820-1825 et, dans certains cas, les années 1830-1835. Elle est aussi annoncée dans une cinquantaine d'inventaires dont nous disposons pour les années 1800-1806. Par conséquent, jusqu'à preuve du contraire et à cause même des éléments de confirmation partielle produits par divers travaux, nous pouvons présumer que l'échantillon, tout imparfait qu'il soit, est représentatif et satisfaisant pour les usages pour lesquels nous l'avons retenu.

\section{Patrimoines fonciers dans la région de Québec (1790-1835)}

Nos débuts d'analyse des patrimoines fonciers à Québec procèdent en deux étapes: d'abord, une description de la structure et de l'évolution

\footnotetext{
articles, il aurait compris ce qui s'y trouve énoncé explicitement: les étendues de terre possédées par les marchands de Montréal et de Québec, par exemple, se situent hors des villes, d'où la distinction très nette que nous avons faite entre ces superficies et les lots dans les villes. Quant aux animaux, Ouellet se réfère à d'autres périodes et à des recensements d'ailleurs fort lacunaires (voir R. M. McInnis, "Some Pitfalls [...]», 221 ss; idem, "A Reconsideration of the State of Agriculture $[\ldots] » ;$ S. Courville, «Villages et agriculture $[\ldots] », 6 \mathrm{ss})$. D'ailleurs, les moyennes constituées à partir de recensements ne mesurent pas forcément la même réalité que celle que captent les inventaires qui, eux, ne traitent que de communautés concrètes et d'exploitations individuelles bien identifiées. Nos divers articles ont spécifié à qui s'appliquaient les inventaires après décès et en quelles circonstances on y recourt. En particulier, ils touchent les communautés ou familles qui ont des enfants. Le professeur Ouellet connait trop le droit coutumier canadien et l'historiographie française de même que les auteurs cités dans le présent article - pour feindre l'ignorance subite à ce propos. Pour la méthodologie, voir note 44. Enfin, Ouellet ne commente guère les deux dernières périodes retenues $(1820-1825,1830-1835)$, pourtant capitales; il ressent toutefois un malaise à nous voir comparer une période de difficulté et une période prospère (17921796 et 1807-1812). Il s'agit d'un reproche paradoxal pour qui connaît l'oeuvre de cet historien: on nous permettra d'enregistrer avec satisfaction sa reconnaissance explicite que la «cassure brutale» de 1802 ne semble plus aussi déterminante et que la grave crise agricole d'après 1802 n'aurait eu que des effets bien courts et moins désastreux que ceux conjecturés dans ses textes antérieurs. 44 Voir, entres autres, les appendices de notre article de 1976. Il a fallu une année pour préciser l'échantillon. Les documents de travail énumèrent tous les inventaires repérés dans les dépôts d'archives, pour toutes les sous-régions et les sous-périodes.
} 
des patrimoines fonciers entre 1790 et 1835; puis, une recherche de corrélation entre certains attributs ou caractéristiques des communautés et la taille de leurs patrimoines fonciers.

\section{A. La structure des patrimoines fonciers}

La reconstruction du patrimoine foncier d'une communauté consiste à agréger les terres (plus de 60 arpents), lopins de terre (de 20 à 60 arpents), parcelles (5-20 arpents) et emplacements ou lots (moins de 5 arpents) énumérés dans l'inventaire après décès. Pour simplifier et raccourcir les intitulés, dans le présent article, nous télescoperons ces catégories en «terres et lopins». L'analyse de ces agrégats peut permettre de discerner des différences structurelles importantes, dans le temps et l'espace. Aussi avons-nous examiné la composition des «patrimoines fonciers» des habitants en excluant les communautés qui ne possèdent rien et celles dont le patrimoine est complètement inconnu ${ }^{45}$. Le tableau 1 présente la structure des patrimoines fonciers pour les sous-régions

\section{TABLEAU 1}

Patrimoines fonciers des habitants, 1792-1835, ventilés par nombre de terres ou lopins (en excluant ceux qui ne possèdent pas de terre), rive nord (RN) et rive sud (RS), en

pourcentages.

\begin{tabular}{|c|c|c|c|c|c|c|c|c|c|c|c|c|}
\hline \multirow{3}{*}{$\begin{array}{c}\text { SOUS- } \\
\text { PÉRIODES }\end{array}$} & \multicolumn{10}{|c|}{ NOMBRE DE TERRES OU LOPINS } & \multicolumn{2}{|c|}{$\begin{array}{l}\text { NOMBRE } \\
\text { D'OBSER- } \\
\text { VATIONS }\end{array}$} \\
\hline & \multicolumn{2}{|c|}{1} & \multicolumn{2}{|r|}{2} & \multicolumn{2}{|r|}{3} & \multicolumn{2}{|c|}{4} & \multicolumn{2}{|r|}{5} & & \\
\hline & RN & RS & $\mathrm{RN}$ & RS & RN & RS & RN & RS & $\mathrm{RN}$ & RS & RN & RS \\
\hline $1792-1796$ & 36,6 & 77 & 32,7 & 11,5 & 9,6 & 7,7 & 9,6 & 0 & 11,5 & 3,8 & & 26 \\
\hline $1807-1812$ & 28,3 & 58,3 & 26,7 & 27,8 & 8,3 & 11,1 & 10 & 0 & 26,7 & 2,8 & 60 & 36 \\
\hline $1820-1825$ & 25 & 82,4 & 12,5 & 11,7 & 22,5 & 0 & 10 & 5,9 & 30 & 0 & 40 & 17 \\
\hline $1830-1835$ & 18,8 & 68,4 & 15,6 & 10,5 & 18,8 & 5,3 & 6,2 & 5,3 & 40,6 & 10,5 & 32 & 19 \\
\hline
\end{tabular}

nord et sud, dans chacune des quatre sous-périodes. Cette structure diffère considérablement d'une rive à l'autre. Sur la rive sud, dans la grande majorité des cas, les habitants ne possèdent qu'une terre, assez grande par ailleurs (voir tableau 2). Même en 1807-1812, cette proportion ne tombe pas en-dessous de 58,3\%, comparativement à un sommet

45 Nous voudrions remercier Serge Courville de son aide dans la clarification de notre présentation de ces divers types d'exploitations agricoles. Même si nos analyses n'ont pas retenu ici toutes les distinctions qu'un chercheur en géographie rurale s'imposerait, elles en ont été considérablement enrichies. Pour ce qui est des patrimoines de dimensions inconnues, nous ne pouvions que les ignorer. Par contre, dans l'analyse du tableau 1, il est possible d'inclure des habitants dont le nombre de terres est connu, même si l'on ignore la superficie exacte de certaines d'entre elles: en effet, le tableau ne se penche pas sur les superficies, mais sur le nombre de terres et de lopins pour chaque communauté. 
de $82,4 \%$ en $1820-1825$. Toutefois, les propriétaires de quatre, cinq terres ou lopins et plus, insignifiants en nombre et en proportion dans les premières sous-périodes, s'accroissent de façon significative en 18301835 (à 15,8\%). Sur la rive nord, la terre unique constitue nettement une situation moins commune, voire de moins en moins importante, relativement, avec le temps. Par contre, au moins le tiers des habitants possèdent deux ou trois terres ou lopins, avec une croissance très marquée, avec les années, de la proportion des communautés disposant de cinq terres ou lopins et plus (de 11,5\% à 40,6\%).

Cette différenciation systématique entre la rive nord et la rive sud peut témoigner d'une intégration plus grande au marché dans la région avoisinant la ville de Québec et l'île d'Orléans, ainsi que d'une zone occupée depuis plus longtemps, plus densément peuplée et plus fertile que le territoire couvert par notre échantillon sur la rive sud. Cependant, même si le patrimoine paraît plus fragmenté sur la rive nord, il comporte le plus souvent une terre principale avec la maison et les bâtiments, sorte de noyau du patrimoine total. Il resterait à déterminer, par une analyse fine, si les habitants propriétaires de plusieurs terres optent pour le faire-valoir direct, familial, ou la location-fermage, etc.

Il convient donc de nuancer la notion de patrimoine foncier pour notre propos: il ne s'agit pas de la transmission d'une seule et même étendue de terre de génération en génération (bien qu'il puisse en être ainsi, notamment sur la rive sud), mais plutôt de celle d'une exploitation d'une étendue moyenne considérable, mais qui peut comprendre plusieurs terres ou lopins obtenus par héritage, donation, achat, saisie, etc. ${ }^{46}$

\section{B. La taille des patrimoines fonciers}

Le tableau 1 laisse soupçonner une certaine accumulation du capital foncier qui se traduit par la proportion de plus en plus élevée, avec le temps, des propriétaires de multiples terres et lopins. Mais cette progression doit aussi être confrontée aux tailles moyennes des exploitations au cours de chaque sous-période. Le tableau 2 précise cette superficie moyenne pour l'ensemble des habitants des rives nord et sud.

La croissance économique rapide de la première décennie de modernisation au 19e siècle a laissé des traces évidentes. Mais dès les années 1820-1825 et par la suite se dessinent un tassement, puis une stabilisation à des niveaux malgré tout élevés par rapport aux moyennes

\footnotetext{
46 Pour scruter cette question plus à fond, il faudrait reconstruire les familles et, à l'aide des terriers, cadastres, actes notariés, etc., pister les lopins et les terres ainsi que les familles qui leur sont liées. Comme le montre le tableau 2, on peut affirmer que même en 1830-1835, au coeur de la «crise agricole», le patrimoine moyen demeure important et ne confirme pas l'hypothèse de la division catastrophique des terres.
} 
TABLEAU 2

Superficie moyenne des exploitations en arpents par inventaire après décès pour les habitants du district de Québec: rive nord/rive sud (1790-1835)*

\begin{tabular}{|cccc|}
\hline $\begin{array}{c}\text { Sous- } \\
\text { périodes }\end{array}$ & $\begin{array}{c}\text { Habitants } \\
\text { rive nord }\end{array}$ & $\begin{array}{c}\text { Habitants } \\
\text { rive sud }\end{array}$ \\
\hline $1792-1796$ & $($ a) & (b) & \\
$1807-1812$ & 99 & 104 & 70 \\
$1820-1825$ & 134 & 146 & 160 \\
$1830-1835$ & 126 & 133 & 88 \\
& 123 & 119 & 94 \\
\hline
\end{tabular}

* L'évaluation (b) comprend des habitants de l'île d'Orléans dont les superficies ont été estimées ainsi qu'on l'a expliqué dans la section 4.A. Nombre d'observations par colonne: 1792-1796: 39, 50, 27; 1807-1812: 44, 57, 36; 1820-1825:39, 42, 16; 1830-1835: 27, 29, 19.

de la fin du $18 \mathrm{e}$ siècle ${ }^{47}$. La taille des exploitations tend à être plus grande sur la rive nord que sur la rive sud (hormis pour les années 18071812). C'est un peu comme si le procès d'accaparement des terres avait commencé plus tôt sur la rive nord, au contact d'un marché plus proche et plus accueillant. Par ailleurs, les superficies moyennes infirment l'hypothèse d'exploitations trop subdivisées, lieux de misère, mais non celle de l'existence d'un prolétariat sans terre dans les campagnes: les recensements nominatifs et des données plus nourries sur les mouvements de population pourraient nous renseigner davantage sur ce dernier point.

Pour cerner d'un peu plus près l'étendue moyenne des véritables exploitations agricoles, nous avons exclu des moyennes les communautés possédant peu ou pas de terre, tant en ce qui a trait à l'ensemble de la population (tous ou «T»), y compris les habitants, qu'en ce qui concerne les habitants seuls $(\mathrm{H})$. Ces «habitants sans terre» sont, la plupart du temps, des personnes qui exercent un autre métier ou qui habitent un village ou qui sont avancées en âge et ont déjà disposé de leurs propriétés, voire d'une bonne partie de leurs biens meubles. Pour identifier ces exploitations agricoles véritables, nous avons défini trois sous-groupes de propriétaires (ayant chacun 2 composantes: $\mathrm{T}$ et $\mathrm{H}$ seulement): 1) les communautés qui possèdent au moins 1 arpent et plus; 2) celles qui en ont au moins 10 et plus; 3 ) enfin, celles qui détiennent au moins 20 arpents et plus ${ }^{48}$. Le tableau 3 retrace une évolution

47 Si la superficie moyenne des terres détenues à la campagne par les divers groupes qui vivent dans la ville de Québec est modeste (pour les quelques exceptions, voir note 40), son évolution suit le même pattern que celle des lots et des maisons de ville: en gros, croissance jusqu'en 1820-1825, puis repli à un niveau cependant supérieur à celui des années 1792-1796.

48 Toute étendue de terre inférieure à 1 arpent, voire à 5 arpents de superficie, est un lot ou emplacement, presque toujours dans une ville ou un village. McInnis estime qu'il faut au moins 10 arpents pour qu'une terre soit «legitimate», alors que Y. Roby et J. Hamelin (Histoire économique et sociale du Québec, 1851-1896 (Montréal, 1971) retiennent plutôt une limite inférieure de 20 arpents. Voir «Some Pitfalls [...]», 222. 
TABLEAU 3

Superficie moyenne des exploitations agricoles (tous - T, habitants - H), 1792-1835, en arpents selon trois catégories de superficie*

\begin{tabular}{|c|c|c|c|c|c|c|c|c|c|c|c|c|}
\hline \multirow[t]{3}{*}{$\begin{array}{c}\text { Sous- } \\
\text { périodes }\end{array}$} & \multicolumn{4}{|c|}{1 arpent ou plus } & \multicolumn{4}{|c|}{10 arpents ou plus } & \multicolumn{4}{|c|}{20 arpents ou plus } \\
\hline & \multicolumn{2}{|c|}{ (a) } & \multicolumn{2}{|c|}{ (b) } & \multicolumn{2}{|c|}{ (a) } & \multicolumn{2}{|c|}{ (b) } & \multicolumn{2}{|c|}{ (a) } & \multicolumn{2}{|c|}{ (b) } \\
\hline & $\mathrm{T}$ & $\mathrm{H}$ & $\mathrm{T}$ & $\mathrm{H}$ & $\mathrm{T}$ & $\mathrm{H}$ & $\mathrm{T}$ & $\mathrm{H}$ & $\mathrm{T}$ & $\mathrm{H}$ & $\mathrm{T}$ & $\mathrm{H}$ \\
\hline $1792-1796$ & 93 & 96 & 97 & 100 & 97 & 99 & 101 & 103 & 105 & 106 & 108 & 109 \\
\hline $1807-1812$ & 154 & 150 & 158 & 155 & 159 & 152 & 163 & 157 & 161 & 154 & 164 & 158 \\
\hline $1820-1825$ & 156 & 122 & 162 & 127 & 167 & 129 & 173 & 134 & 167 & 129 & 173 & 134 \\
\hline $1830-1835$ & 126 & 124 & 124 & 122 & 134 & 127 & 131 & 125 & 136 & 130 & 134 & 127 \\
\hline
\end{tabular}

* Les colonnes (a) excluent les 5 propriétaires de 2000 arpents et plus (voir note 40 ) et les communautés pour lesquelles nous ne disposons pas de données fermes concernant la superficie de leurs terres. Les colonnes (b) incluent des communautés de l'île d'Orléans pour lesquelles une estimation minimale des superficies a été tentée. Pour les quatre sous-périodes et selon les colonnes (a) et (b), le nombre d'observations retenues est le suivant par ordre:

1792-1796: $63,60,74,71 ; 60,58,71,69 ; 55,54,66,65$

1807-1812: 91, 79, 104, 92; 88, 78, 101, 91; 87, 77, 100, 90

$1820-1825: 59,52,63,55 ; 55,49,59,52 ; 55,49,59,52$

1830-1835: 48, 41, 50, 43; 45, 40, 47, 42; 44, 39, 46, 41 
des superficies moyennes analogue à celle inscrite dans le tableau 2, sauf que ces superficies, dans les exploitations agricoles véritables, demeurent élevées même en 1830-1835. Alors que l'ensemble des groupes occupationnels grossissent leurs patrimoines jusqu'en 18201825 inclusivement, les habitants enregistrent un repli net, mais non catastrophique, dès cette troisième sous-période et sont rejoints par les autres catégories en 1830-1835. En fait, les niveaux de richesse foncière moyenne se sont accrus entre la fin du $18 \mathrm{e}$ siècle et la fin du premier tiers du 19e siècle. Par ailleurs, il est clair qu'une analyse plus fine devra étudier la morphologie des exploitations (arpents cultivés, en jachère, en bois debout, etc.) et son changement au cours de la période.

\section{Différenciation croissante des patrimoines fonciers et accaparement du sol}

La hausse, puis la stabilisation ou le repli relatif de la taille moyenne des patrimoines fonciers, se conjuguent à une différenciation socioéconomique croissante des habitants que nous avions déjà signalée ailleurs ${ }^{49}$. Ce processus se déploie tout au long des trois premières décennies du 19e siècle (tableau 4). La proportion des habitants sans terre (villageois, personnes plus âgées, locataires, etc.), semble stable entre 1792-1796 et 1830-1835. En fait, elle tombe dans les seconde et troisième périodes, puis elle grandit en 1830-1835. La proportion des propriétaires de très grandes étendues ( 200 arpents et plus) bondit entre la première et la dernière sous-période.

Par ailleurs, le pourcentage de propriétés modestes (de 1 à 89 arpents) tout comme celui des patrimoines moyens ou grands (de 90 à 199 arpents) chutent avec le temps, érodés par les deux extrêmes: respectivement de $41 \%$ à $33 \%$ et de $42 \%$ à $39 \%$, après une poussée à $54 \%$ en 1807-1812.

Cette différenciation socio-économique, au niveau des patrimoines fonciers, transparaît encore plus clairement lorsqu'on examine l'accaparement du sol par les très gros habitants - ceux qui possèdent 200 arpents et plus (tableau 5) ${ }^{50}$. Avant le décrochage du début du 19e siècle, de 6 à $7 \%$ des communautés rurales accaparent environ le cinquième des terres; dans les années 1830 , ce sont $17 \%$ qui ont accumulé

49 G. Paquet et J.-P. Wallot, «Les inventaires après décès $[\ldots]$, «Structures sociales et niveaux de richesse $[\ldots] »$, «Groupes sociaux et pouvoir [...]»; «Sur quelques discontinuités dans l'expérience socio-économique du Québec: une hypothèse», $R H A F, 35,3$ (décembre 1982): 483521 .

50 Il semble que les marchands et les membres des professions libérales de la ville de Québec ne s'approprient guère une part importante des avoirs fonciers dans les campagnes environnantes. Quelques rares individus possèdent de très vastes étendues de terre dans les cantons. Quoi qu'il en soit, les moyennes d'arpents par gros habitants accapareurs ne diffèrent pas beaucoup de celles des gros propriétaires urbains. 
TABLEAU 4

Répartition de la propriété foncière des habitants (1792-1835)*

\begin{tabular}{|c|c|c|c|c|c|c|c|c|}
\hline \multicolumn{9}{|c|}{ SUPERFICIE DES EXPLOITATIONS (EN ARPENTS) } \\
\hline $\begin{array}{c}\text { Sous } \\
\text { périodes }\end{array}$ & 0 & $1-49$ & $50-89$ & $90-149$ & $150-199$ & $200-399$ & $400-999$ & 1000 et plus \\
\hline $\begin{array}{l}1792-1796 \\
\text { (a) } \\
\text { (b) }\end{array}$ & $\begin{array}{l}6(9,1) \\
6(7,8)\end{array}$ & $\begin{array}{l}14(21,2) \\
14(18,2)\end{array}$ & $\begin{array}{l}13(19,7) \\
17(22)\end{array}$ & $\begin{array}{l}25(37,9) \\
29(37,7)\end{array}$ & $\begin{array}{l}3(4,5) \\
6(7,8)\end{array}$ & $\begin{array}{l}4(6,1) \\
4(5,2)\end{array}$ & $\begin{array}{l}1(1,5) \\
1(1,3)\end{array}$ & \\
\hline $\begin{array}{l}1807-1812 \\
\text { (a) } \\
\text { (b) }\end{array}$ & $\begin{array}{l}4(4,8) \\
4(4,2)\end{array}$ & $\begin{array}{l}8(9,6) \\
8(8,3)\end{array}$ & $\begin{array}{l}14(16,9) \\
16(16,7)\end{array}$ & $\begin{array}{l}26(31,3) \\
29(30,2)\end{array}$ & $\begin{array}{l}20(24,1) \\
25(26,0)\end{array}$ & $\begin{array}{r}9(10,8) \\
11(11,5)\end{array}$ & $\begin{array}{l}1(1,2) \\
2(2,1)\end{array}$ & $\begin{array}{l}1(1,2) \\
1(1,0)\end{array}$ \\
\hline $\begin{array}{l}1820-1825 \\
\text { (a) } \\
\text { (b) }\end{array}$ & $\begin{array}{l}3(5,4) \\
3(5,2)\end{array}$ & $\begin{array}{l}10(18,2) \\
10(17,3)\end{array}$ & $\begin{array}{l}9(16,4) \\
9(15,5)\end{array}$ & $\begin{array}{l}21(38,2) \\
22(37,9)\end{array}$ & $\begin{array}{l}6(10,9) \\
6(10,3)\end{array}$ & $\begin{array}{l}4(7,3) \\
6(10,3)\end{array}$ & $\begin{array}{l}2(3,6) \\
2(3,5)\end{array}$ & \\
\hline $\begin{array}{l}1830-1835 \\
\text { (a) } \\
\text { (b) }\end{array}$ & $\begin{array}{l}5(10,9) \\
5(10,4)\end{array}$ & $\begin{array}{l}8(17,4) \\
9(18,7)\end{array}$ & $\begin{array}{l}7(15,2) \\
7(14,6)\end{array}$ & $\begin{array}{l}15(32,6) \\
16(33,3)\end{array}$ & $\begin{array}{l}3(6,5) \\
3(6,3)\end{array}$ & $\begin{array}{l}7(15,2) \\
7(14,6)\end{array}$ & $\begin{array}{l}1(2,2) \\
1(2,1)\end{array}$ & \\
\hline
\end{tabular}

* Les données de ce tableau excluent les superficies inconnues et celles de plus de 2000 arpents. La série (b) tient compte cependant d'estimés minimaux pour certaines terres de l'île d'Orléans. Nombre d'exclusions:

1792-1796: (a) 18, (b) 7; 1807-1812: (a) 19, (b) 6;

1820-1825: (a) 4, (b) 1; 1830-1835: (a) 6, (b) 4 .

Les données entres parenthèses représentent les pourcentages de la répartition pour la période. 
$40 \%$ des terres du groupe. Les étendues moyennes croissent sensiblement pour revenir, en 1830-1835, à un niveau moyen légèrement supérieur à celui de la fin du $18 \mathrm{e}$ siècle.

\section{TABLEAU 5}

Accaparement du sol par les habitants (1792-1835): habitants avec 200 arpents et plus*

\begin{tabular}{|lccccc|}
\hline \multicolumn{1}{|c}{$\begin{array}{c}\text { Sous- } \\
\text { périodes }\end{array}$} & $\begin{array}{c}\text { Nombre de } \\
\text { ménages }\end{array}$ & $\begin{array}{c}\% \mathrm{du} \\
\text { total }\end{array}$ & $\begin{array}{c}\text { nombre } \\
\text { d'arpents }\end{array}$ & $\begin{array}{c}\% \mathrm{du} \\
\text { total }\end{array}$ & $\begin{array}{c}\text { moyenne } \\
\text { d'arpents } \\
\text { par ménage }\end{array}$ \\
\hline $1792-1796$ & & & & & \\
(a) & 5 (sur 66) & 7,6 & 1304 & 22,6 & 261 \\
(b) & 5 (sur 77) & 6,5 & 1304 & 18,3 & 261 \\
$1807-1812$ & & & & & \\
(a) & 11 (sur 83) & 13,3 & 4398 & 37,1 & 400 \\
(b) & 14 (sur 96) & 14,6 & 5418 & 37,9 & 387 \\
$1820-1825$ & & & & & \\
(a) & 6 (sur 55) & 10,9 & 2140 & 33,9 & 357 \\
(b) & 8 (sur 58) & 13,8 & 2703 & 38,6 & 338 \\
$1830-1835$ & & & & & \\
(a) & 8 (sur 46) & 17,4 & 2132 & 41,8 & 266 \\
(b) & 8 (sur 48) & 16,7 & 2132 & 40,7 & 266 \\
\hline
\end{tabular}

* Pour le nombre de données non retenues, voir la note au bas du tableau 4 .

\section{TABLEAU 6}

Taille des exploitations (en arpents) et alphabétisation, 1792-1835 (habitants seulement)*

\begin{tabular}{|lcccc|}
\hline \multicolumn{1}{|c}{$\begin{array}{c}\text { Sous- } \\
\text { périodes }\end{array}$} & alphabètes & $\begin{array}{c}\text { nombre de } \\
\text { ménages }\end{array}$ & $\begin{array}{c}\text { nombre de } \\
\text { ménages }\end{array}$ \\
\hline $\begin{array}{l}1792-1796 \\
\text { a) }\end{array}$ & 85 & $(8)$ & 88 & $(58)$ \\
(b) & 94 & $(10)$ & 92 & $(66)$ \\
$1807-1812$ & & & & \\
(a) & 283 & $(11)$ & 124 & $(73)$ \\
(b) & 270 & $(15)$ & 127 & $(81)$ \\
$1820-1825$ & 141 & & & \\
$($ a) & 156 & $(13)$ & 107 & $(42)$ \\
(b) & & $(14)$ & 109 & $(44)$ \\
$1830-1835$ & 118 & $(18)$ & 109 & \\
(a) & 117 & $(19)$ & 107 & $(28)$ \\
(b) & & & & $(29)$ \\
\hline
\end{tabular}

* Pour l'explication des séries (a) et (b), voir tableau 3. 


\section{Alphabétisation et capital foncier}

$\mathrm{Si}$, comme le suggère la section 3.C du présent texte, les habitants poursuivent une stratégie d'accumulation du capital foncier, il devrait être possible d'établir un rapport entre certaines caractéristiques des ménages inventoriés et l'importance de leur patrimoine foncier. Ainsi, aux États-Unis, les études de Newell sur l'Ohio, au début du 19e siècle, montrent que l'alphabétisation des familles constitue une variable significative dans l'explication de la croissance des fortunes. En un sens, l'alphabétisation correspond ceteris paribus à une qualité additionnelle du capital humain susceptible de conduire à une stratégie économique plus réussie.

Pour vérifier cette conjecture, le tableau 6 compare la superficie moyenne du patrimoine foncier des ménages dont le survivant sait signer, à celle des familles où ce survivant ne le sait pas, cette capacité de signer servant ici de critère pour départager les alphabétisés des analphabètes. Si la différence entre les deux types de ménages est négligeable à la fin du $18 \mathrm{e}$ siècle, l'alphabétisation devient une variable significative au $19 \mathrm{e}$ siècle et joue, pour toutes les sous-périodes, dans le sens d'une superficie plus étendue. Le degré d'alphabétisation des habitants, tel qu'il ressort des inventaires, dépasse les moyennes habituellement avancées par l'historiographie ${ }^{51}$. Le fait de demeurer près de Québec, du marché et d'écoles peut rendre compte de cet écart: une analyse similaire pour l'aire géographique plus étendue de la région de Montréal devrait déprimer ces pourcentages.

\section{E. Nombre d'enfants et capital foncier}

Plus significatif encore dans le procès d'accumulation du capital foncier devrait être le nombre des enfants. En effet, si, comme le propose la section 3.C, les habitants - et d'autres groupes sociaux, sans doute - aspirent à établir leurs enfants, un plus grand nombre d'enfants devrait donc les inciter à accroître leur patrimoine. Simultanément, ces enfants plus nombreux constituent, pour un ménage donné, un capital humain additionnel susceptible de faciliter l'accumulation et l'exploitation d'un capital foncier plus considérable ${ }^{52}$.

Les données du tableau 7 corroborent notre conjecture dans tous les cas (sauf 1792-1796 pour les ménages de 9 enfants et plus). Une corrélation positive entre le nombre d'enfants et la taille moyenne du patrimoine foncier s'impose nettement. Or elle est d'autant plus impor-

51 Voir A. Greer, «L'alphabétisation et son histoire au Québec: état de la question», in L. Lamonde, prés., L'imprimé au Québec. Aspects historiques (18e-20e siècles) (Québec, 1983), 77-125.

R. C. On trouvera des ébauches de cette hypothèse dans les travaux de L. Michel, L. Dechêne, R. C. Harris et A. Barthez. Pour l'Ohio, Newell note aussi qu'il y a corrélation positive entre le niveau de richesse et le nombre d'enfants. W. H. Newell, op.cit, 110-111. 
TABLEAU 7

Taille des exploitations (en arpents) et nombre des enfants, 1792-1835

(habitants seulement)*

\begin{tabular}{|lccccc|}
\hline \multicolumn{1}{c}{$\begin{array}{c}\text { Sous- } \\
\text { périodes }\end{array}$} & \multicolumn{5}{c|}{ Nombre d'enfants } \\
\hline & 0 & $1-4$ & $5-8$ & 9 et plus & Inc. \\
\hline $1792-1796$ & - & $74(37)$ & $109(25)$ & & \\
(a) & - & $82(45)$ & $108(27)$ & $102(5)$ & 7 \\
(b) & & & & & \\
$1807-1812$ & $75(1)$ & $115(44)$ & $137(32)$ & $165(4)$ & 8 \\
(a) & $75(1)$ & $117(48)$ & $151(38)$ & $161(6)$ & \\
(b) & - & $90(25)$ & $112(23)$ & $120(4)$ & 5 \\
$1820-1825$ & - & $90(25)$ & $116(25)$ & $120(4)$ & \\
(a) & & & & & \\
(b) & - & $85(21)$ & $120(17)$ & $148(6)$ & 7 \\
$1830-1835$ & - & $85(21)$ & $120(17)$ & $148(6)$ & \\
(a) & & & & & \\
(b) & & & & & \\
\hline
\end{tabular}

* Les chiffres entre parenthèses indiquent le nombre d'observations. Les inconnus comprennent les communautés pour lesquelles on ne connaît pas la superficie du patrimoine ou encore le nombre d'enfants (l'inventaire parle parfois d'«enfants mineurs» et de «tuteur» et «subrogé tuteur» sans autre spécification).

tante qu'on a trop souvent réduit les familles nombreuses à un indicateur socio-économique de retard culturel et de mauvais planning familial. A tout le moins, une interprétation de rechange se pointe et là aussi, semble-t-il, les observateurs ont peut-être conclu trop rapidement à l'irrationnalité de comportements qui, à l'analyse, se révèlent raisonnables et économiquement rentables. Par ailleurs, une étude plus poussée qui prendrait en compte l'âge des enfants, leur sexe, etc., éclairerait sans doute davantage le sens de la corrélation relevée.

\section{F. Richesse immobilière et richesse mobilière}

On peut s'interroger également sur une autre forme de corrélation: y a-t-il un lien positif de complémentarité entre la richesse immobilière et la richesse mobilière, telles qu'établies à partir des inventaires, ou constituent-elles des formes de richesse de substitution? Le tableau 8 ventile les habitants selon qu'ils possèdent une superficie de terre supérieure ou inférieure à la taille moyenne des patrimoines de leur région (rive nord et rive sud séparément), pour chaque sous-période. Il fournit les résultats d'une confrontation entre le statut de chaque habitant par rapport aux niveaux moyens de richesse immobilière et mobilière ${ }^{53} \mathrm{de}$

53 Par richesse mobilière, nous entendons tous les biens meubles à l'exclusion des espèces, métaux précieux, dettes actives et dettes passives. Nous les avons répartis, ailleurs, en 10 catégories (G. Paquet et J.-P. Wallot, «Les inventaires après décès [...]», appendice I et $208 \mathrm{ss})$. La richesse mobilière nette ajoute (ou soustrait) aux 10 catégories le solde de la monnaie, des métaux et des dettes. 


\section{TABLEAU 8}

Richesse mobilière et richesse immobilière des habitants, 1792-1835*

\begin{tabular}{|c|c|c|c|c|}
\hline \multirow{3}{*}{$\begin{array}{l}\text { Ventilation des habitants en } \\
\text { groupes selon que leur } \\
\text { richesse immobilière est plus } \\
\text { grande ou plus petite que la } \\
\text { moyenne }(\overline{\mathrm{x}})\end{array}$} & \multicolumn{4}{|c|}{$\begin{array}{l}\text { Probabilité conditionnelle que la richesse mobilière } \\
\text { observée est plus grande ou moins grande que la moyenne } \\
\text { (x) pour un niveau de richesse immobilière donnée }\end{array}$} \\
\hline & \multicolumn{2}{|c|}{ rive nord } & \multicolumn{2}{|c|}{ rive sud } \\
\hline & $<\overline{\mathrm{x}}$ & $>\overline{\mathrm{X}}$ & $<\overline{\mathrm{x}}$ & $>\overline{\mathrm{x}}$ \\
\hline \multicolumn{5}{|l|}{$1792-1796$} \\
\hline$<\overline{\mathrm{x}}$ & 76,2 & 23,8 & 76,9 & 23,1 \\
\hline$>\overline{\mathrm{x}}$ & 27,8 & 72,2 & 64,3 & 35,7 \\
\hline \multicolumn{5}{|l|}{$1807-1812$} \\
\hline$<\tilde{\mathrm{x}}$ & 86,4 & 13,6 & 80,8 & 19,2 \\
\hline$>\overline{\mathrm{x}}$ & 40,9 & 59,1 & 30,0 & 70,0 \\
\hline \multicolumn{5}{|l|}{$1820-1825$} \\
\hline$<\overline{\mathrm{x}}$ & 83,3 & 16,7 & 80,0 & 20,0 \\
\hline$>\overline{\mathrm{x}}$ & 40,0 & 60,0 & 54,5 & 45,5 \\
\hline \multicolumn{5}{|l|}{$1830-1835$} \\
\hline$<\overline{\mathrm{X}}$ & 81,3 & 18,7 & 69,2 & 30,8 \\
\hline$>\bar{x}$ & 36,4 & 63,6 & 33,3 & 66,7 \\
\hline
\end{tabular}

* On peut lire ce tableau de la façon suivante (colonnes de gauche, $1^{\text {ère }}$ ligne): si on examine le sous-groupe d'habitants de 1792-1796 sur la rive nord qui ont moins de terre que la moyenne des habitants de la rive nord durant cette période, la probabilité que la richesse mobilière d'un habitant tiré au hasard dans ce groupe soit plus faible que la moyenne de la richesse mobilière du groupe est de $76,2 \%$; la probabilité que sa richesse mobilière soit plus grande que la moyenne est de $23,8 \%$.

sa sous-région, de façon à déterminer si sa richesse est supérieure ou inférieure à la moyenne: en d'autres mots, il s'agit de voir si l'habitant a une richesse mobilière et des avoirs immobiliers qui tous deux sont au-dessus ou en-dessous de la moyenne.

Il se dégage une forte corrélation entre ces deux formes de richesse pour toutes les périodes et les deux sous-régions, à deux exceptions près (ceux qui détiennent une fortune immobilière plus grande que la moyenne sur la rive sud en 1792-1796 et en 1820-1825). Mais dans 14 cas sur 16, la corrélation s'établit, ce qui vient étayer notre hypothèse d'un lien étroit entre les deux types de richesse. Bref, le pattern dominant, c'est celui du gros exploitant, bien nanti en biens meubles et pouvant compter sur plusieurs enfants, ou, à l'inverse, la communauté moins bien dotée à tous égards et avec un nombre restreint d'enfants ${ }^{54}$.

54 La composante ethnique, utilisable pour la ville de Québec, n'est d'aucune utilité comme variable dans les campagnes. Dans tout l'échantillon, il n'y a qu'un seul habitant anglo-saxon. Y. Morin a trop généralisé à partir du cas de la ville de Québec («La représentativité de l'inventaire après décès $[\ldots] \gg)$. 
Si le tableau 8 avait retenu la richesse mobilière nette, c'est-à-dire tous les biens meubles ainsi que les dettes «actives» et «passives», la corrélation positive demeurerait, mais à un niveau moins fort et avec un peu plus d'exceptions. La raison en apparaît clairement à la lecture des inventaires individuels: il arrive à des habitants de s'endetter pour acquérir des terres additionnelles - ou les conserver - au lieu de sacrifier des biens meubles, de sorte que leur richesse mobilière nette (y compris les effets financiers) est moins clairement reliée à la taille du patrimoine foncier que la richesse en biens meubles proprement dits.

Dans le cas présent, il n'a pas semblé nécessaire de recourir aux séries (a) et (b), sur la rive nord, vu qu'elles sont généralement concordantes. Seule la série (a) a servi à l'élaboration du tableau.

$$
* * *
$$

Les données sur les patrimoines fonciers corroborent largement nos conjectures quant à l'existence d'une stratégie foncière chez les habitants du Bas-Canada, au tournant du 19e siècle: stratégie qui privilégie ce secteur d'activité et qui manifeste une rationalité économique que l'historiographie traditionnelle a eu trop souvent tendance à nier. Non seulement l'habitant arrondit-il son patrimoine foncier, mais l'évolution de la structure du capital foncier décalque celle d'une structure socio-économique en procès de différenciation dans la région de Québec. A côté d'agriculteurs plus à l'aise et plus nombreux qui accaparent davantage de terres, se forme un groupe de paysans sans terre qui doivent se rabattre sur les villages et les villes. Des communautés plus alphabétisées, avec plus d'enfants, tendent à consolider des patrimoines fonciers plus étendus.

Voilà autant d'éléments susceptibles de fonder une interprétation de rechange du comportement de l'habitant. Plus besoin, maintenant, d'invoquer le chromo puisque les faits semblent étayer le modèle d'un habitant proactif, que nous avons proposé plus haut.

\section{Conclusion}

Si notre hypothèse paraît vraisemblable, à la lumière des données, il faudra passer au crible une multitude de mythes et d'actes de foi trop répandus dans l'historiographie, générale et spécialisée, et tous ancrés dans une vision chromotique et même chromophile de l'habitant. Un nouveau pas pourrait ainsi être franchi, avec le renvoi ou la révision de certaines grandes interprétations qui continuent à hanter les manuels et à nourrir la facilité. C'est plutôt vers des révisions assez radicales qu'il faut s'orienter dans plusieurs dossiers considérés comme clos dans l'histoire socio-économique du Québec. La mythologie a trop longtemps bloqué la recherche. 
Pour notre part, depuis 1967, nous avons amorcé cette nécessaire remise en question pour le début du $19 \mathrm{e}$ siècle, comme d'autres le font pour des périodes antérieures ou plus longues, tels Pierre Tousignant, Louise Dechêne, Louis Michel, Jacques Mathieu, Serge Courville, ou pour des temps plus récents, tels Paul-André Linteau, Jean-Claude Robert, Normand Séguin, John McCallum, Gérard Bouchard, etc. ${ }^{55}$ En particulier, il faudra réouvrir le dossier des Rébellions de 1837-1838 et chercher à composer une interprétation moins strictement culturelle, idéologique et politique (derrière des étiquettes souvent «sociales») au sens restreint, mais davantage enracinée dans les phénomènes économiques. Ce travail est déjà commencé, mais beaucoup reste à faire ${ }^{56}$. De la même façon, l'histoire rurale du Québec commence à être réécrite selon des schèmes d'interprétation fort différents des anciens clichés ${ }^{57}$. Finalement, il se pourrait qu'une économie politique des institutions, s'échafaudant sur un modèle de comportement stratégique de l'habitant, suggère des versions nouvelles des grands débats politiques du 19 e siècle ${ }^{58}$.

En attendant que s'explicitent ces réinterprétations, le chromo demeure vivace et encombrant, malgré tous les travaux qui en ont déjà souligné le caractère inadéquat. C'est que comme toutes les images d'Epinal, celle-ci revêt une simplicité rassurante. Le jour où l'on osera admettre que cette simplification et les raccourcis qu'elle a inspirés sont aussi peu fondés que les autres raccourcis racistes ou sexistes qu'on a extirpés de nos manuels depuis quelques décennies, il se pourrait qu'une vigoureuse reconstruction de l'histoire économique de l'habitant sur des bases moins friables permette enfin à l'historiographie canadienne de réaliser la révolution que les Américains ont déjà accomplie en ce domaine il y a déjà bien longtemps.

\section{ANNEXE I}

\section{L'habitant: une radiographie économique des deux hypothèses}

Dans le langage des économistes, les différences de comportement observées peuvent avoir leur source soit dans des patterns de préfé-

55 Nous avons cité plusieurs travaux de ces historiens. Concernant la transmission des patrimoines à une époque plus récente, voir G. Bouchard, «Les systèmes de transmission des avoirs familiaux et le cycle de la société rurale au Québec du XVIIe au XXe siècle», Histoire sociale, 16 (1983): 35-60.

56 Voir G. Paquet, «Une économique du phénomène révolutionnaire», miméo, 90 p. J.-P. Bernard donne un aperçu des interprétations les plus connues et suggère des pistes à explorer dans Les rébellions de 1837-1838 (Montréal, 1983).

57 Notamment dans les travaux récents déjà cités et ceux de N. Séguin: La conquête du sol au 19e siècle (Québec, 1977); Agriculture et colonisation du Québec (Montréal, 1980); «L'agriculture de la Mauricie et du Québec, 1850-1950», RHAF, 35,4 (mars 1982): 537-562; idem et R. Hardy, Forêt et société en Mauricie (Montréal, 1984).

58 Ainsi une réévaluation de tout un pan de notre histoire politique du $19 \mathrm{e}$ siècle pourrait utiliser l'approche dite du «public choice». Pour un exemple de cette approche appliquée au Québec du 19e siècle, voir R. Carter, «Séparation, annexion et fédéralisme: au-delà des préceptes normatifs usuels», L'Actualité économique, 59 (1983): 596-619. 
rences différents de certains groupes d'acteurs, soit dans des différences de l'environnement économique qui les contraint.

La première famille d'explications plus subjectives a été utilisée pour suggérer que l'habitant avait peut-être simplement peu de goût pour le risque. Voilà qui pourrait expliquer un comportement qui minimise le risque systématiquement sous la seule contrainte d'un revenu minimal à obtenir. Attitude qui ferait contraste avec le comportement d'acteurs britanniques qui auraient un goût pour le risque et qui donc chercheraient à maximiser leurs revenus sous la seule contrainte d'un risque qui ne devrait pas dépasser certaines bornes. Dans le langage de l'économiste, cette aversion pour le risque n'est pas irrationnelle et pourrait se traduire dans des logiques de choix (fonctions de préférence ou d'utilité) différentes pour l'habitant et ses concurrents britanniques.

Bien qu'une telle explication ne soit pas nécessairement sans fondements, nous avons pour notre part opté pour la seconde famille d'explications. Nous croyons que c'est plutôt dans la configuration de l'espace des possibilités réelles de l'habitant que dans la configuration de sa fonction de préférence qu'il faut chercher la clé de ses comportements. Les deux sociétés (canadienne et britannique) au Canada ne font pas nécessairement face au même ensemble effectif de possibilités d'investissements à cause de leur accès différentiel à l'information et à la finance. Voilà qui fait que, même avec une aversion similaire pour le risque, il s'ensuit une stratégie d'accumulation différente. Il ne s'agit pas de nier la possibilité que des technologies sociales différentes puissent enclencher des attitudes différentes par rapport au risque (le manque de réseaux familiaux étendus, capables de servir de filet de sécurité économique, peut fort bien par exemple, entraîner l'habitant à avoir une propension moins grande à prendre des risques); mais il nous semble que le gros de l'explication tient aux conditions objectives auxquelles l'habitant et ses concurrents font face. Ces conditions sont différentes. Les deux types d'explication sont illustrés dans les graphiques suivants:

L'espace FC représente en ordonnée la propriété foncière et en abscisse la propriété commerciale. Dans le graphique I, les possibilités d'investissements auxquelles font face les deux groupes (habitant: $h$; britannique: b) sont définies comme identiques et représentées par l'espace concave OXX'; l'espace OXX', définit le champ des possibilités d'investissements fonciers ou commerciaux ouvert et accessible à l'un ou l'autre des groupes; tout point hors OXX', est hors d'atteinte et tout point à l'intérieur du bloc OXX' ne tire pas tout le parti possible de ces possibilités; tous les points sur la frontière $\mathrm{XX}$ ' sont des points également efficaces. Les fonctions de préférence ou d'utilité des deux groupes $\left(U_{h}\right.$ et $\left.U_{b}\right)$ diffèrent cependant et sont représentées par des courbes convexes incorporant un différentiel d'intérêt pour les deux formes d'investissement - un différentiel d'aversion pour le risque par exem- 


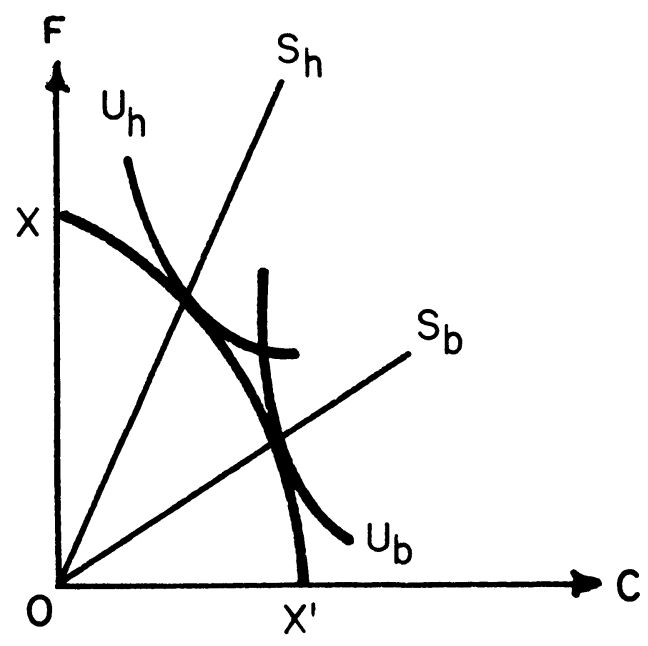

Graphique I

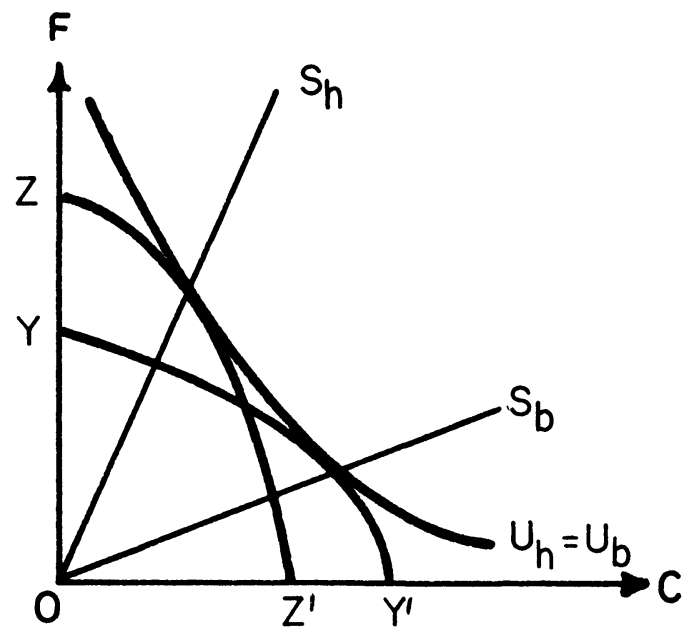

Graphique II 
ple - Elles représentent un niveau d'iso-utilité pour le groupe: le lieu d'ensembles de $\mathrm{F}$ et $\mathrm{C}$ qui laissent le groupe également satisfait. On peut montrer que l'optimum est atteint quand la fonction de préférence est tangente au bloc des possibilités. A ce point de tangence, le groupe atteint le plus haut niveau de satisfaction compatible avec les frontières du bloc de possibilités. Dans le graphique II, les possibilités objectives d'investissements diffèrent entre les deux groupes (OZZ' pour les habitants et OYY' pour les Britanniques), à cause de leur accès différentiel à l'information et à la finance, mais les fonctions de préférence sont identiques. On voit qu'il n'est pas nécessaire de postuler des mentalités ou des motivations ou des aversions pour le risque différentes pour les deux groupes (comme on le fait au graphique I), pour obtenir des stratégies différentes d'accumulation. Les vecteurs $S_{\mathrm{h}}$ et $S_{\mathrm{b}}$ sont utilisés pour indiquer la direction générale des stratégies d'accumulation des deux groupes, dans le contexte des deux situations décrites par les graphiques I et II. Ces vecteurs montrent, dans les deux cas, une stratégie qui met l'accent sur la propriété foncière dans le cas des habitants et une stratégie qui met l'accent sur la propriété commerciale dans le cas des Britanniques, mais le fondement de ces choix repose sur des raisons différentes. Dans le cas du Graphique I, cette stratégie différenciée prend sa source dans les préférences ou «mentalités» différentes des deux groupes alors que dans le graphique II, cette stratégie différenciée prend sa source dans la nature différente des possibilités effectives d'investissements. Il est évidemment possible que les comportements différents observés soient attribuables à un mélange des deux ensembles de forces; mais avant d'avoir recours aux différences de mentalités, il faut, il nous semble, chercher à comprendre les écarts de comportement objectivement attribuables à des contextes effectivement différents. 


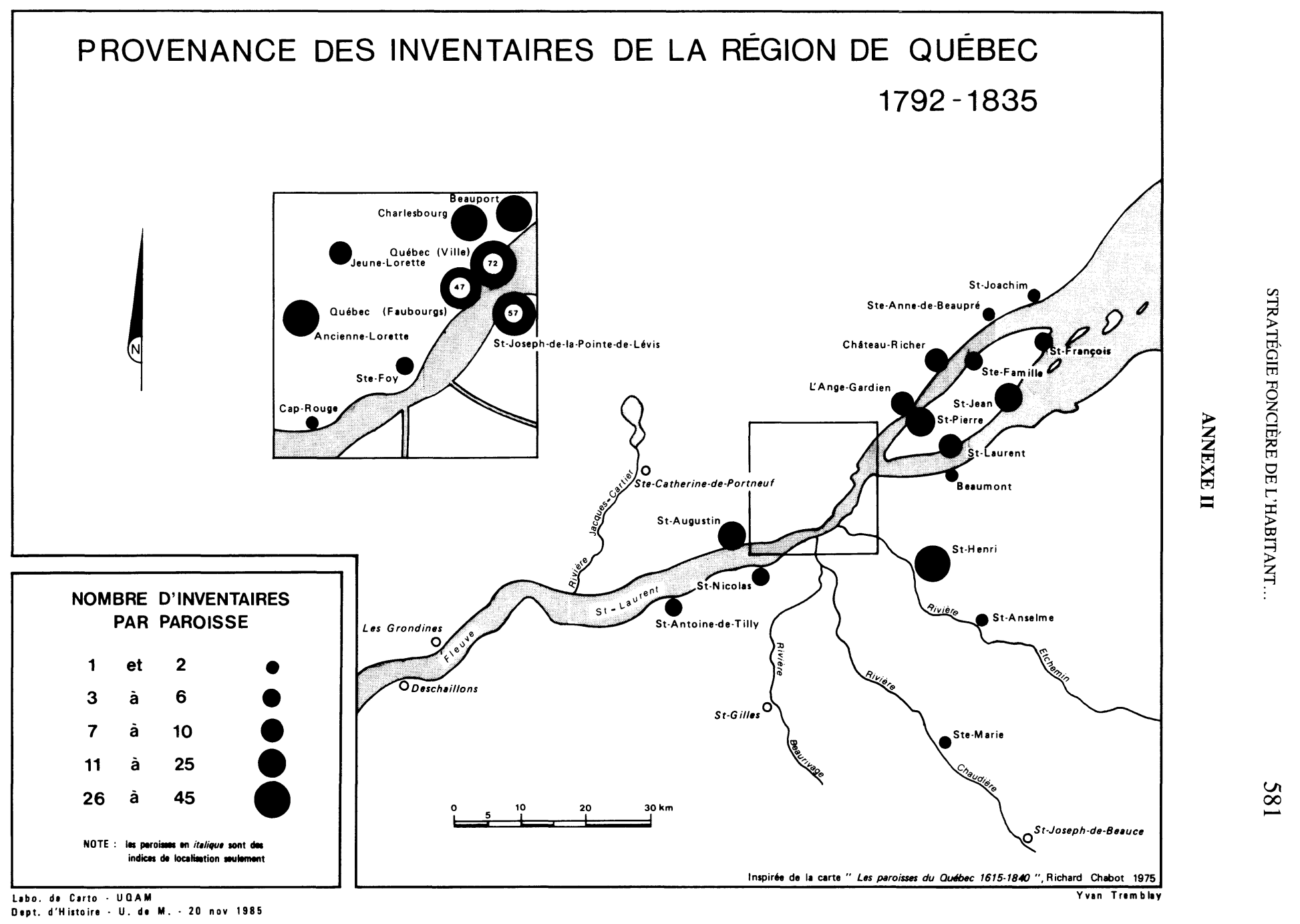

